\title{
La Encuesta Socio Ambiental: instrumento para el estudio integrado de espacios naturales protegidos. Aplicación a la Sierra Sur de Sevilla y Sierra de Cádiz
}

\section{The Socio-Environmental Survey: a tool for an integrated study of protected areas. The case of Sierra Sur de Sevilla and Sierra de Cádiz}

\author{
Marta F. Calvache ${ }^{1}$, Fernando Díaz del Olmo², \\ Rafael Cámara ${ }^{3}$ y Eva B. Rodríguez ${ }^{4}$
}

\begin{abstract}
RESUMEN
La declaración de Espacios Naturales Protegidos ha producido con frecuencia conflictos con las poblaciones locales en la gestión de la conservación de la naturaleza. En respuesta a esta realidad han surgido nuevos métodos y estrategias que buscan una mejor integración de la componente social en las áreas protegidas. Este artículo presenta los resultados de una Encuesta Socio-Ambiental (ESA) realizada durante la elaboración de una propuesta de Espacio Natural Protegido para la Red de Espacios Naturales Protegidos de Andalucía en la campiña agrícola del Subbético, entre las provincias de Sevilla y Cádiz (sur de España). A partir de los datos obtenidos se propone
\end{abstract}

${ }^{1}$ Investigadora, marta.fdez.calvache@gmail.com. ORCID iD: http://orcid.org/0000-00027575-3926.

2 Profesor Catedrático, Departamento de Geografía Física y Análisis Geográfico Regional, Universidad de Sevilla, delolmo@us.es. ORCID iD: http://orcid.org/0000-0002-0658-4989.

${ }_{3}^{3}$ Profesor Titular, Departamento de Geografía Física y Análisis Geográfico Regional, Universidad de Sevilla, rcamara@us.es. ORCID iD: http://orcid.org/0000-0003-1046-3749.

${ }^{4}$ Investigadora de Doctorado, Departamento de Geografía Física y Análisis Geográfico Regional, Universidad de Sevilla, rodriguezperez.eva@gmail.com. ORCID iD: http://orcid.org/ 0000-0002-7813-4039. 
y discute la aplicación de la figura de Paisaje Protegido (tipo Categoría V de UICN) asociada a un modelo de gestión participativa abierta.

PAlabras Clave: espacios naturales protegidos; encuesta socio-ambiental; agrosistemas; paisajes protegidos; gestión participativa.

\begin{abstract}
The declaration of Protected Areas has frequently led to conflicts with local populations because of nature conservation management. In response to this reality new methods and strategies are emerging that are looking for a better integration of the social component in Protected Areas. This article shows the results of a Socio-Environmental Survey (SES) conducted during the elaboration of a Protected Area proposal for the Andalusian Protected Areas Network in the Subbetic agricultural "campiña", between Seville and Cádiz provinces (Southern Spain). Based on the data obtained, the application of the Protected Landscape figure (type Category V of IUCN) associated to an open participatory management model is proposed and discussed.
\end{abstract}

KEY WORDS: protected areas; socio-environmental survey; agro-systems; protected landscapes; participatory management.

Cómo citar este artículo / Citation: Calvache, Marta F., Díaz del Olmo, Fernando, Cámara, Rafael y Rodríguez, Eva B. (2017): "La Encuesta Socio Ambiental: instrumento para el estudio integrado de espacios naturales protegidos. Aplicación a la Sierra Sur de Sevilla y Sierra de Cádiz", Estudios Geográficos, LXXVIII/282, pp. 31-69.

\title{
I. INTRODUCCIÓN
}

En las últimas décadas las políticas de conservación de la naturaleza in situ, o sea, las relacionadas con el establecimiento de espacios naturales protegidos (ENPs) han experimentado importantes cambios. España no ha estado al margen de ellos como se evidencia al contrastar los cambios legislativos desde 1916, año de la primera Ley de Parques Nacionales, con el conjunto de leyes posteriores, incluyendo las autonómicas, hasta el actual 2016 (Morillo y Gómez-Campo, 2000). Como ha sintetizado Mace (2014), se ha pasado de una conservación de la naturaleza en sí misma, o sea declarando ENPs «intocables» para la acción antrópica, esto es, el modelo de «reservas» aisladas (Abakerli, 2001; Barber et al., 2004; Toledo, 2005; Guevara y Laborde, 2008; Gurrutxaga San Vicente y Lozano Valencia, 2009), a otra en la que las actividades económicas están presentes o incluso configuran la base de un nuevo modelo de desarrollo socio-económico caracterizado como sostenible, que llega hasta conectar las estrategias de conservación con la reducción de la pobreza 
(Lausche, 2011). A nivel internacional la expresión más genuina de esta transformación son el aumento considerable de las Reservas de Biosfera del Programa MaB de la UNESCO y la propuesta de la Categoría V (Paisaje Protegido) de la UICN. En España y en Europa su aplicación se ha hecho a través de la figura de Parque Natural que comienza a utilizarse a principios de los años ochenta en respuesta a las nuevas formas de ecodesarrollo (Díaz del Olmo y Molina Vázquez, 1985; Colón y Díaz del Olmo, 1989), paso previo a las acciones de sostenibilidad derivadas de la Conferencia de las Naciones Unidas sobre el Medio ambiente y el Desarrollo en Río de Janeiro (1992) y la adopción del Convenio sobre la Diversidad Biológica (CDB) (Furze et al., 1996).

Así pues, a partir de mediados de la primera década del siglo XXI, las figuras nacionales de conservación tipo Categoría V de la UICN (Parque Natural, Parque Regional, o algunos casos de Reservas Naturales, Paisajes Protegidos o incluso de Áreas Marinas Protegidas), unido a la relevancia para la conservación de las acciones de desarrollo sostenible, determinan el giro actual hacia las manifestaciones del paradigma de los ENPs en territorios antropizados pero sensibles a la conservación del medio natural (Locke y Dearden, 2005). En ellos el planteamiento principal es incorporar la planificación y gestión de la conservación, sin que genere oposición poblacional (Troitiño Vinuesa et al., 2005), o en todo caso, minimizando el rechazo de la declaración de un espacio y desactivando los conflictos sociales en las comunidades que se sientan afectadas (Geoghegan y Renard, 2002; Zimmerer, 2006). Su importancia radica en el hecho de que la mayor parte de la conservación in situ se hace en territorios no-wild, donde son necesarias la continuidad de las prácticas tradicionales para la conservación de determinados hábitats y especies. En consecuencia, el nuevo modelo favorece la incorporación de novísimos elementos del patrimonio natural para la conservación "clásica», tales como la agro-diversidad y la geoconservación (Martino, 2005; Mallarach et al., 2008; Carcavilla et al., 2009; Gray, 2013).

Por su parte, cómo abordar la conflictividad social se ha convertido en un fin en sí mismo para las políticas de conservación en la actualidad. Una respuesta ha venido de las propuestas de interpretación de los ENPs como Sistemas Socio-Ecológicos (SSE) (Folke et al., 1996; Toledo, 2005; Gómez Vargas et al., 2008; Quiroga, 2009; Martín-López et al., 2011; Sarkar y Montoya, 2011), donde la participación pública de los pobladores y usuarios locales (stakeholders) se integre en las formas de gestión con presencia real, no sólo en las fases informativas, sino en las «tomas de decisión» de las diferentes etapas de la planificación y gestión (adaptativa, co-gestión, co-desarrollo, manejo comunitario) (Galvin y Haller, 2008; Gómez Baggethun, 2009; Sosa et al., 2014). El objetivo mayor es hacer realidad a través de la conservación algunas 
de las vías que favorecen el alcance de la denominada sostenibilidad social (Wallance et al., 2011). Son estas las prácticas de la conocida New Conservation Science (en adelante NCS) que impulsa a la vez la integración de los actores y saberes locales, la adecuada participación, la valorización de los servicios ecosistémicos y los valores éticos de los ENPs. Expresado en síntesis, la NCS trata de superar el debate tradicional parks vs. people (Minteer y Miller, 2011; Miller et al., 2011; Sarkar y Montoya, 2011; Soulé, 2013), y postula una conservación en pie de igualdad nature and people (Mace, 2014).

En la actualidad la superficie terrestre protegida en España se encuentra próxima al 28\%, considerando los ENPs amparados por la Ley 42/2007 de Patrimonio Natural y de la Biodiversidad (que representan aproximadamente el $12,5 \%$ de la superficie protegida), los de la Red Natura, así como los designados por otros instrumentos internacionales (MAGRAMA, 2015). Vinculadas a esta superficie, habría que añadir las áreas de influencia socioeconómica (municipios total o parcialmente incluidos en un parque), que en 2005 fueron estimadas por Troitiño Vinuesa et al. (2005) en un 40\% del territorio nacional. Lo que constata el creciente protagonismo territorial de los espacios protegidos bajo alguna de las figuras legales existentes, implicando extensas áreas de interacción entre la conservación y el resto de usos del territorio.

\section{II. ¿Espacios Naturales Protegidos en territorios agropecuarios? Objetivo y ÁREA DE ESTUDIO}

En el presente artículo, acorde con la NCS y las propuestas de la sostenibilidad social, se plantea una figura de ENP para una propuesta territorial de conservación de la naturaleza in situ. Esta se propone con base en los resultados de la aplicación de una Encuesta Socio-Ambiental (en adelante ESA), presentados en las siguientes secciones, a través de la que se busca obtener información sobre la percepción y saberes de los problemas medio ambientales y socio económicos de la población local, entendida como actores locales presentes y futuros para una gestión participativa y adaptativa coherente con una figura legal protectiva y con las actividades que impliquen sostenibilidad social de los recursos.

Su aplicación se lleva a cabo en un conjunto de municipios de la Sierra Sur de Sevilla y Sierra de Cádiz, en el sur de España, dada la calidad ambiental de su patrimonio natural, sus paisajes, la continuidad de sus agrosistemas, y la existencia de recursos naturales como parte importante del desarrollo económico de los municipios, donde un grupo de actores locales se ha movilizado en torno a una iniciativa cultural y educativa de extensión universitaria deno- 
minada Aula Miguel Cala Sánchez (AMCS) radicada en Morón de la Frontera (Sevilla) ${ }^{5}$ con apoyo de profesores de los centros educativos locales y de investigadores de la Universidad de Sevilla.

El área de estudio abarca $1.204 \mathrm{~km}^{2}$ correspondiendo a los municipios de Alcalá del Valle, Algámitas, Algodonales, Cañete la Real, Coripe, El Gastor, El Saucejo, Montellano, Morón de la Frontera, Olvera, Pruna, Puebla de Cazalla, Puerto Serrano, Torre Alháquime y Villanueva de San Juan, en el límite sureste de la provincia de Sevilla, norte de Cádiz y noroeste de Málaga (figura 1).

Desde el punto de vista paisajístico forma parte de la campiña alta del valle del Guadalquivir, así como de los macizos y peñones del Subbético Externo de la Cordillera Bética. Desde una perspectiva hidrográfica es el territorio donde se instalan las cabeceras de las cuencas del Guadaíra y Corbones, ambos afluentes del Guadalquivir por la margen izquierda, y del Guadalete. La hipsometría del relieve fluctúa entre los $1.100 \mathrm{~m}$. del Terril en la Sierra del Tablón (Sevilla) y los $160 \mathrm{~m}$. del río Guadaíra al pie de la Sierra de Esparteros (Morón de la Frontera, Sevilla). A nivel bioclimático, el área de estudio constituye una transición de los pisos bioclimáticos Termomediterráneo y Mesomediterráneo inferior, caracterizado por temperaturas media de verano de $18-20^{\circ} \mathrm{C}$ y de invierno de $16^{\circ} \mathrm{C}$, con una pluviometría muy variable interanualmente entre 600 y $1.000 \mathrm{~mm}$ anuales. Entre las formaciones vegetales autóctonas dominan: los encinares y alcornocales adehesados en su gran mayoría con cortejo de coscoja (Quercus coccifera), lentisco (Pistacia lentiscus), palmitares (Chamaerops humilis) y jarales basófilos dominantes (Cistus albidus), restos de quejigal (Quercus faginea) en áreas de montaña con cortejo de cornicabras (Pistacia terebinthus); y en las riberas de ríos y arroyos olmedas (Ulmus minor) y tarajales (Tamarix gallica y T. africana). En la matriz antrópica del paisaje domina como cultivo el olivar. En la fauna destaca una importante concentración de rapaces representadas principalmente por núcleos de anidamiento de buitres leonados (Gyps fulvus) y la presencia del alimoche (Neophron percnop-

${ }^{5}$ El Aula Miguel Cala Sánchez es una iniciativa de Extensión Universitaria puesta en marcha en 1998, radicada en la localidad de Morón de la Frontera para el ámbito comarcal de la Sierra Sur de Sevilla y entorno de las sierras de Málaga y Cádiz, patrocinada por el Vicerrectorado de Relaciones Institucionales de la Universidad de Sevilla. Para el desarrollo de sus actividades funciona autónomamente con apoyos económicos e institucionales solicitados periódicamente. Mantiene dos tipos de campos de actuación en relación con la conservación del patrimonio: el natural y el histórico-cultural. Para el desarrollo de sus actividades el Aula aglutina actores locales (empresarios, agricultores, ganaderos, profesores locales, alumnos de la ESO y Bachillerato, estudiantes y profesores universitarios, asociaciones de jubilados y mujeres, representantes de partidos políticos, ecologistas y de los ayuntamientos y diputaciones). 
FIGURA 1

MAPA DE LOCALIZACIÓN

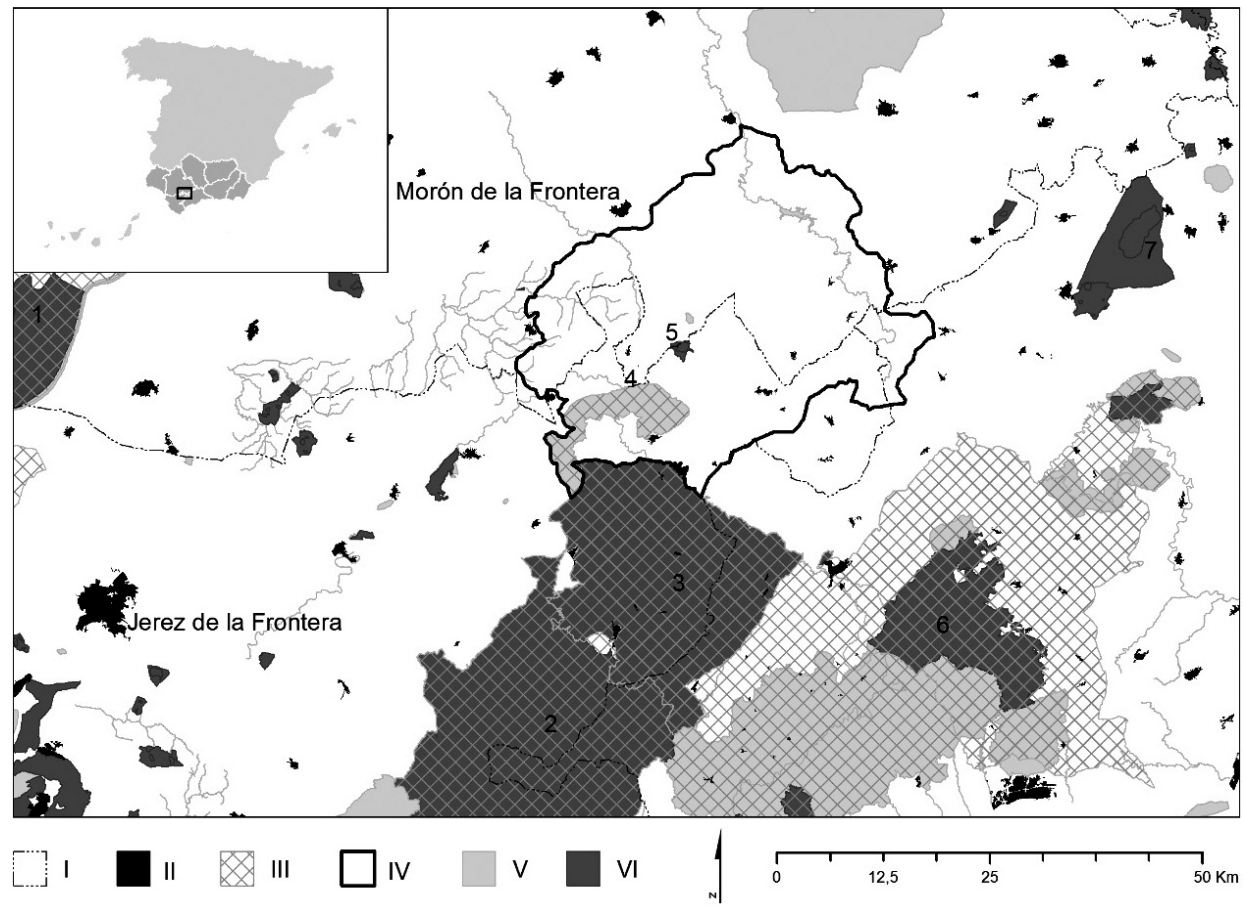

Fuente: base de datos de la Red de Información Ambiental de Andalucía, REDIAM .

(I: Límites administrativos. II: Núcleos de población principales. III: Reserva de la Biosfera. IV: Propuesta de protección. V: Espacios Red Natura 2000. VI: Espacios Naturales protegidos. 1. Parque Nacional Doñana; 2. Parque Natural Los Alcornocales; 3. Parque Natural Sierra de Grazalema; 4. Monumento Natural El Chaparro de la Vega; 5. Reserva Natural Peñón de Zaframagón; 6. Parque Natural Sierra de las Nieves; 7. Reserva Natural Laguna de Fuente de Piedra).

terus), el águila perdicera (Aquila fasciata), y el búho real (Bubo bubo) en el Peñón de Zaframagón, y las colonias de cernícalo primilla (Falco naumanni) en los peñones calizos sobre todo los Peñones de Olvera. El territorio del área de estudio se emplaza estratégicamente en un entramado regional de ENPs, representando una continuación natural de los hábitats, formaciones geomorfológicas y formas de vida, al norte y noroeste de los Parques Naturales de Sierra de Grazalema, Sierra de las Nieves, Los Alcornocales, todos ellos Reserva de la Biosfera, que a su vez configuran las áreas núcleo de la Reserva de la Biosfera Intercontinental del Mediterráneo, de la que forman parte también las áreas protegidas del norte de Marruecos. 
La población del área es 89.653 personas según el último censo realizado (2011). En el momento de realizar el trabajo el dato utilizado fue el del padrón municipal de 2008, que aportaba un total de 89.783 habitantes. A pesar de atravesar una importante crisis, en 2003 la agricultura es el principal uso del suelo ( $50 \%$ según el mapa de usos y coberturas de Andalucía) y actividad económica. Se trata, por lo tanto, de un área altamente dependiente del sector agropecuario, con escaso desarrollo de sectores económicos alternativos y con un alto grado de dependencia de subvenciones de la PAC a la actividad y al trabajador agrícola. En definitiva, se trata de un clásico paisaje antropizado que en Andalucía se conoce como «campiñas», modelado por las actividades económicas históricas del sector primario.

El área de la propuesta de protección (figura 1) fue inicialmente delimitada con base en un estudio biogeográfico. Dentro de su perímetro fueron designadas dos áreas diferenciadas, correspondientes a una zona de protección ecológica y otra de usos compatibles, que pasaremos a referir como zonas central y periférica. La primera de estas, en la que vive el $73 \%$ de la población, englobaría las áreas de alto valor ambiental, cultural y paisajístico, con bajo nivel de transformación antrópica, incluyendo además de los territorios más naturales, zonas de dehesas y pastizales. Por su lado, la periférica incluiría aquellas zonas de fuerte carácter agrario, teniendo no obstante un estatus de mayor protección las áreas con función de conectividad, amortiguamiento o restauración.

La propuesta de ENP planteada por el AMCS se inició como una oferta abierta al debate e información en los ámbitos municipales de la comarca perfilada a lo largo de los años de trabajo colectivo con dos grandes etapas. La primera abarcó una amplia red de municipios del territorio interprovincial a la cual se refiere este trabajo, realizada entre 2000-2010, cuyo objetivo fue hacer un levantamiento de información territorial con catálogos de flora, fauna, georrecursos, bienes culturales, datos históricos de archivos y socioeconómicos, etc. Sus resultados se presentaron y discutieron en talleres y congresos del AMCS. Al final de este período corresponde el levantamiento de la ESA que se presenta en este artículo. La segunda etapa, 2011-2016, se ha orientado a la catalogación, sistematización, confección de cartografía y apoyo de una propuesta de conservación de los paisajes, recursos naturales y culturales del área de estudio, junto a la elaboración de algunas iniciativas locales para la dinamización de actividades socio-económicas alternativas y complementarias de la comarca.

El resultado principal del AMCS ha sido elevar y defender ante las Delegaciones Provinciales de Sevilla y Cádiz de la Consejería de Medio Ambiente de la Junta de Andalucía y a su Dirección General de Espacios Protegidos, y paralela- 
mente ante el Parlamento de Andalucía una Proposición No de Ley (PNL), de una propuesta de ENP, de Paisaje Protegido conforme a la ley 1989 de Espacios Naturales de Andalucía. En la actualidad (2016) todavía no se ha producido ninguna declaratoria de ENP.

\section{MATERIALES, MÉTODOS Y TÉCNICAS}

\section{La ESA: cuestionario y tratamiento estadístico}

Desarrollamos la ESA como herramienta para la realización del estudio exploratorio para la propuesta de conservación in situ, durante la fase de ordenación de los recursos y diagnóstico territorial, por permitir un levantamiento de información socio-económica que proyecta la percepción del territorio de sus habitantes. La información obtenida ha sido integrada junto a los datos estadísticos, cartográficos y biogeográficos.

\section{Cuestionario: fases y características}

La elaboración del cuestionario se llevó a cabo tomando en consideración dos objetivos finalistas: (1) Evaluar la receptividad poblacional hacia la potencial declaración de un ENP en la comarca; y (2) analizar las necesidades de desarrollo territorial sostenible desde el punto de vista de la población y actores locales.

El formato elegido tiene dos fases. En la primera se elabora un pre-cuestionario obtenido a partir de la fase de levantamiento de información. Este se compuso de 34 preguntas y se ejecutó en octubre de 2009, a través de una muestra de 38 encuestados (14\%).

En la segunda fase se elaboró el cuestionario definitivo compuesto por 31 preguntas, tras la corrección de anomalías, simplificación del vocabulario, reestructuración y acortamiento del cuestionario, inclusión de opciones adicionales y eliminación de cuestiones ambiguas, redundantes o irrelevantes, o ausencias en las preguntas y respuestas del pre-cuestionario.

La encuesta definitiva fue realizada a lo largo de 7 jornadas de campo distribuidas entre los meses de febrero, marzo y septiembre de 2010. Para ello se contó con equipos de 2 a 4 entrevistadores. Si bien la recogida de datos en diferentes periodos podría introducir alteraciones en los resultados, se consideró que el corto periodo de tiempo transcurrido entre las campañas no supondría una variación sustancial en las percepciones de los encuestados. 
Sobre el terreno, los entrevistados eran elegidos aleatoriamente, siguiendo no obstante los criterios establecidos en cuanto a sector de ocupación, sexo y edad. Fueron así recogidos un total de 261 cuestionarios, superando en 4 la muestra prevista.

El resultado final para el trabajo de la ESA es un cuestionario cerrado anónimo a realizar de forma personal e individual, rellenado por el entrevistador e implementado en los municipios total o parcialmente incluidos en el área de estudio.

\section{Tratamiento estadístico}

El muestreo realizado fue de tipo no probabilístico por cuotas, calculadas a partir de datos de ocupación del censo de 2001 (en ese momento el más reciente a nivel municipal), usando como unidad administrativa de base los municipios incluidos total o parcialmente en el área de estudio. La encuesta se dirigió a la población rural local, entendiendo como la principal protagonista de una gestión participativa en una declaración de un espacio protegido. Por ello, la población universo fue calculada de modo que contuviese un $70 \%$ de población agraria y un 30\% de población ocupada en otros sectores (en base a los datos de ocupación del censo de 2001), ya que la población agraria sería la más afectada ante la declaración de un espacio protegido. Fue usada como base la variable «Población en viviendas familiares ocupada de 16 y más años clasificada por actividad, según municipio de residencia», a cuyos valores fueron adicionados un $30 \%$ de población ocupada en otros sectores, resultando en una población universo de 10.190 habitantes. Desafortunadamente el censo de 2011 no produjo datos de ocupación por sectores a nivel municipal, con los que habría sido posible contrastar los cambios en el perfil ocupacional de la población. No obstante, el censo de 2011 muestra un incremento de un 2,2\% de la población total sobre el censo de 2001 de los municipios parcial o totalmente incluidos en el área de estudio, principalmente en los de la zona central. Por lo que se considera que estos datos y el corto periodo de tiempo transcurrido desde la realización de la encuesta justifican la validez actual de los resultados.

Para el cálculo de la muestra, fueron usadas las siguientes ecuaciones:

$\mathrm{n}^{\prime}=\mathrm{S}^{2} / \mathrm{V}^{2}$

$\mathrm{n}=\mathrm{n}^{\prime} /\left(1+\mathrm{n}^{\prime} / \mathrm{N}\right)$

Donde (n') representa el tamaño provisional de la muestra, (n) el tamaño corregido de la muestra, $\mathrm{N}$ la población universo, $\mathrm{S}^{2}$ la varianza de la muestra (siendo $S^{2}=\mathrm{p}(1-\mathrm{p})$ y $\mathrm{p}$ la probabilidad de obtener un resultado $\mathrm{p}$ multiplicado 
por la probabilidad de obtener un resultado 1-p), y $\mathrm{V}^{2}$ la varianza de la población, que equivale al cuadrado del error muestral elegido. Fue considerado un valor de $\mathrm{p}=0,5$ y un error muestral de $3,1 \%$, permitiendo una muestra razonable y adaptada a los medios disponibles. Con base en estos valores, se obtuvo una muestra de encuestados de 254 personas.

Adicionalmente y considerando que los municipios centrales verían sus usos más comprometidos con la eventual declaración de un espacio protegido, se les aplicó un factor multiplicador con el fin de que en ellos fueran realizadas el doble de encuestas. En base a lo anterior, fue calculado el número de encuestas a realizar en cada zona. El reparto de encuestas por municipio se obtuvo en función del peso de la población agraria de cada municipio sobre el tamaño de la población agraria del área central y de la periférica. Posteriormente, estos valores fueron ajustados para garantizar un mínimo de 5 encuestas por municipio que permitiera la significación estadística, resultando en una muestra final de 257 personas. A este respecto, los municipios que quedaban infrarrepresentados con el reparto, fueron agrupados (p.e. El Gastor, Torre Alháquime y Alcalá del Valle, por un lado; Cañete la Real, Algámitas y Villanueva de San Juan por otro). Dentro de cada municipio, las entrevistas fueron distribuidas considerando el reparto por sexos de la población ocupada en los diferentes sectores.

Los cuestionarios recogidos fueron codificados mediante el mismo software con el que se diseñaron (SPSS Data EntryBuilder) y analizados usando el programa de análisis estadístico SPSS, siendo el objetivo principal realizar un estudio descriptivo en base a las frecuencias observadas. En aquellas variables en que el número de casos por categoría fue suficiente para realizar un análisis de correlaciones, fue utilizado el estadístico Chi-cuadrado de Pearson $\left(\chi^{2}\right)$ en una tabla de contingencia bidimensional. Este estadístico parte de la hipótesis de independencia de las variables, contrastando posteriormente las frecuencias esperadas y observadas. De este modo, una probabilidad asociada a $\chi^{2}$ mayor a 0,05 , sirve para confirmar la independencia de las variables, mientras que valores inferiores a 0,05 llevan a rechazar la hipótesis de independencia y a concluir que existe algún grado de relación. De cara a construir variables más robustas y así facilitar un análisis de las tablas de contingencia, dentro de algunas variables las categorías fueron reagrupadas (categorías con menos de 5 casos). A modo de ejemplo, la variable edad, que inicialmente contaba con 4 categorías (20-34, 35-50, 51-64, >0=65), fue reagrupada en 3 clases (20-34, $35-50,>50)$. Con este mismo fin, las respuestas clasificadas como No Sabe/No contesta (NSNC), fueron consideradas como valor perdido, salvo en aquellos casos en los cuales el peso de la opción NSNC supuso un porcentaje sustan- 
cial de las respuestas. Finalmente, debido al bajo número de datos para determinados municipios (algunos contaban con 5 entrevistas), se optó por un análisis global del área de estudio.

\section{Materiales complementarios y cartografía}

De manera específica para este artículo se han utilizado las coberturas cartográficas del Mapa de Usos y Coberturas Vegetales de Andalucía (MUCVA, 2003) de escala 1:25.000 y la base de datos de la Red de Información Ambiental de Andalucía REDIAM (http://www.juntadeandalucia.es/medioambiente/ site/rediam, Consejería de Medio Ambiente y Ordenación del Territorio, Junta de Andalucía) para los mapas vectoriales municipales y límites de la Red de Espacios Naturales Protegidos de Andalucía (RENPA).

\section{APLiCACIÓN DE LA ESA}

Como se ha indicado líneas atrás, el cuestionario de la ESA estuvo dirigido a obtener una información proporcional y adecuada para conocer el grado de receptividad de la población local a la declaración de un ENP en un ámbito geográfico y socio-económico plenamente insertado en la realidad rural de Andalucía Occidental.

La ESA se estructuró en cuatro bloques. Uno hace referencia a la familiaridad con el concepto de ENP, grado de conocimiento de espacios protegidos cercanos, la disposición del encuestado a la conservación y al tipo de gestión más acorde con una propuesta determinada de ENP. Otro bloque está centrado en obtener información sobre la percepción territorial de la población local, específicamente sobre los problemas socioeconómicos y medioambientales que afectan a la comarca y las medidas potenciales para su corrección. Dentro de este apartado se evalúa así mismo el conocimiento de los subsidios a la agricultura, por considerarse este un factor determinante en la actualidad del sistema económico del área de estudio. Un nuevo bloque está orientado a futuros procesos de participación evaluando el sentimiento de pertenencia, las fuentes de acceso a la información, el nivel de asociacionismo y la disposición a la participación pública. Un último bloque, que es el primero en la interpretación de los resultados, está destinado a la caracterización sociodemográfica de la población encuestada, incluyendo variables como edad, procedencia, tiempo de residencia en la zona, sector de ocupación y situación profesional, propiedad de tierras y nivel de estudios. 


\section{Caracteres sociodemográficos}

Al tratarse de un muestreo no probabilístico por cuotas, estaba prevista una mayor participación del género masculino, debido a su predominio en el sector agrario. No obstante, su participación final en el total de la muestra fue del 75,5\%, superando el peso predefinido. Este desequilibrio se debió parcialmente a una mayor reticencia del sexo femenino a la participación en la encuesta.

En total el 74,7\% de los encuestados están en el rango de edad 20-50 años, habiendo nacido la gran mayoría $(91,2 \%)$ en la comarca, y siendo la mayor parte residentes de largo plazo. En el momento de la entrevista, el 35,2\% de los encuestados se encontraba desocupado y la mitad de los mismos en situación de desempleo. El perfil ocupacional de la muestra corresponde a un $46,7 \%$ de individuos pertenecientes al sector primario, un 13\% a la industria y construcción, y un $36,7 \%$ al sector servicios. En cuanto al perfil profesional, estuviesen o no ocupados en aquel momento, un 23,4\% de los participantes eran empresarios o trabajadores autónomos, un $34,1 \%$ asalariados fijos y un $34,9 \%$ asalariados eventuales.

Entre los encuestados dominaban los que carecían de tierra en propiedad $(65,9 \%)$. Dentro de los propietarios, la mitad lo eran de fincas menores de 5 hectáreas, siendo el siguiente grupo más abundante el de 5-20 ha de terreno. Finalmente, y de acuerdo con el nivel educativo, el grupo mayoritario (37,9\% de encuestados), fue el de aquellos con graduado escolar, siendo relevante un $24,1 \%$ sin graduado, y un $13,4 \%$ con estudios universitarios.

\section{Percepción territorial}

Las preguntas principales de este segundo bloque se dirigieron a conocer la opinión de los encuestados sobre la percepción y saberes que tenían acerca de los problemas y las soluciones en el ámbito socioeconómico y medioambiental.

De todos los problemas socioeconómicos, señalados sobre una lista predefinida, destaca la escasez de empleo (92\%), la dependencia del subsidio agrario $(65,1 \%)$ y el bajo precio de los productos agrarios $(64,4 \%)$, siendo común entre todos los entrevistados que recurrieron a la opción 'Otra respuesta' la debilidad de la industria como un asunto acuciante (Anexo I). No es de extrañar que entre las respuestas seleccionadas para la corrección de estos problemas los resultados fueron: la necesidad de creación de empresas (52,9\%), el impulso al turismo rural $(44,1 \%)$ y a la industria agroalimentaria $(43,3 \%)$. Adi- 
cionalmente a las medidas aportadas por el cuestionario, varios entrevistados propusieron como solución el fomento del tejido industrial.

Además del contexto socioeconómico, fue también abordada la cuestión medioambiental local. Para ello se ofreció igualmente una lista de problemas y afecciones, siendo considerados como más importantes por los encuestados la suciedad de los ríos debido a vertidos (53,6\%), el mal estado o invasión de los cauces fluviales $(50,2 \%)$ así como de las vías pecuarias $(49,8 \%)$.

Por su lado, entre las soluciones propuestas más populares para mejorar las condiciones medioambientales del municipio, se hizo referencia a la reforestación y a la mejora de la gestión forestal (51\%), al apoyo a la agricultura ecológica $(47,9 \%)$ y la delimitación y restauración de cauces y vías pecuarias $(38,3 \%)$.

Cuestionados sobre las subvenciones agrícolas, la relevancia de la valoración del producto agrícola constituyó una respuesta mayoritaria (44,8\%) (figura 2), si bien se detectó una falta de información, desconocimiento o desconfianza en materia de la PAC.

FIGURA 2

OPINIÓN SUBVENCIONES AGRÍCOLAS

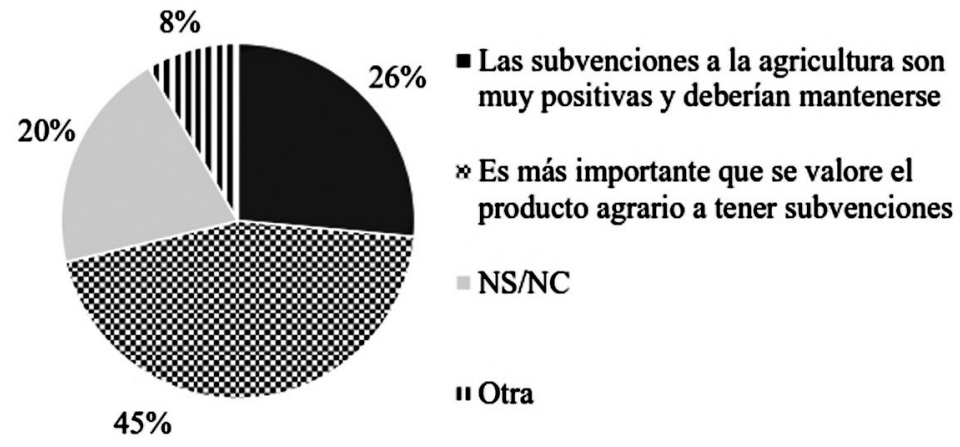

Familiaridad con los espacios protegidos y disposición a la protección

Desarrollo sostenible es un concepto que los encuestados conocen como ponen de manifiesto los resultados obtenidos: $60,5 \%$ ha oído hablar del término y un $47,9 \%$ incluso identifica correctamente su definición. Destaca asimismo una alta valoración del entorno natural; un 44,8\% considera el valor del medio natural de su municipio como alto. Esta valoración resultó mayori- 
taria, independientemente de la ocupación, perfil profesional y nivel educativo de los encuestados. Ciertamente se identifican algunos sesgos de valoraciones más altas en los encuestados del sector servicios y empresarios, así como en la probabilidad asociada a $\chi^{2}$ que relaciona la edad con esta cuestión, siendo más positiva en la franjas de edades mayores de 35 años y entre los propietarios de tierras (Anexo II).

Por su parte el concepto de ENP es ampliamente conocido (91,9\%), mucho más que el de desarrollo sostenible, como hemos indicado anteriormente. El 35\% y el 21,2\% de los conocedores del concepto identifican la función de los ENPs con «regulación de actividades económicas y fomento de la conservación de la naturaleza» o con el impulso a «una economía de menor impacto ambiental y nuevas fuentes de empleo»; y un 20,4\% con acciones limitantes para el desarrollo económico en función de la conservación de la naturaleza (figura 3). Adicionalmente, un $22,6 \%$ de aquellos familiarizados con el término, conocían la figura de protección de tres o más espacios protegidos cercanos al área de estudio. El pequeño porcentaje de los que decían desconocer el concepto, conocían sin embargo en su mayoría la existencia de estos espacios: del 7,3\% que no había oído hablar de ENP, un 89,5\% sí conocía Grazalema o Doñana, un 52,6\% Fuente de Piedra, un 36,8\% El Chaparro de la Vega y un 47,4\% el Peñón de Zaframagón.

FIGURA 3

FUNCIÓN PERCIBIDA DE LOS ENPS

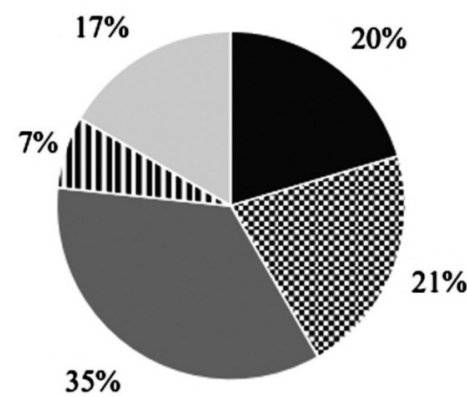

- Establecen prohibiciones y limitan el desarrollo económico para conservar la naturaleza

* Impulsan una economía de menor impacto ambiental y ofrecen nuevas fuentes de empleo

- Regulan las actividades económicas y fomentan la conservación de la naturaleza

" Otra

$=\mathrm{NS} / \mathrm{NC}$

Al someter al análisis de $\chi^{2}$ la cuestión de la identificación de las figuras de protección, se detecta su relación con la tenencia de la tierra. Aquellos que poseen tierras tienen un mayor grado de conocimiento de los espacios circundantes y sus figuras, siendo que el $34,2 \%$ de los propietarios entrevistados 
conocía las figuras de tres o más ENPs, frente a un 17\% de los no propietarios. Los encuestados con menor conocimiento de las figuras de ENPs fueron los del sector primario y asalariados eventuales.

A la pregunta central de este bloque acerca de si consideraban que su municipio o alguna parte del mismo debía ser protegido, planteada a aquellos que afirmaron conocer el concepto de ENP, un 52,5\% respondió afirmativamente, el 25,4\% negativamente y un 10,8\% lo apoyaría con ciertas condiciones.

Con el fin de poder realizar un análisis más robusto de las tablas de contingencia, la variable de disposición a la protección fue reagrupada, quedando por un lado aquellos que respondieron afirmativamente a la protección y por otro, los que se opusieron o bien asintieron con condiciones. Se hizo evidente la existencia de correlación entre esta variable y el sector de ocupación, siendo el apoyo a la protección más reducido en el sector primario. Un 41,5\% de los encuestados del primario, un 59,4\% de los del sector de la industria y la construcción y un 59,8\% de los ocupados en el sector servicios, apoyaron la opción de proteger. El estadístico $\chi^{2}$ demostró asimismo la existencia de relación entre la disposición a proteger y la tenencia de la tierra.

A aquellos que optaron por la protección y a los que dieron su apoyo con condiciones, se les presentaron diferentes opciones de formas de gestión, al objeto de obtener una respuesta sobre las características del tipo de figura que mejor se adaptaría a la propuesta de conservación (figura 4). Las medidas más apoyadas fueron una gestión basada en el turismo rural y deportivo (46\%), en la conservación de fauna y flora $(39,5 \%)$, las acciones agroganaderas $(31,6 \%)$ y la protección y conservación de recursos naturales (28,9\%).

\section{FIGURA 4}

TIPO DE GESTIÓN DESEADA

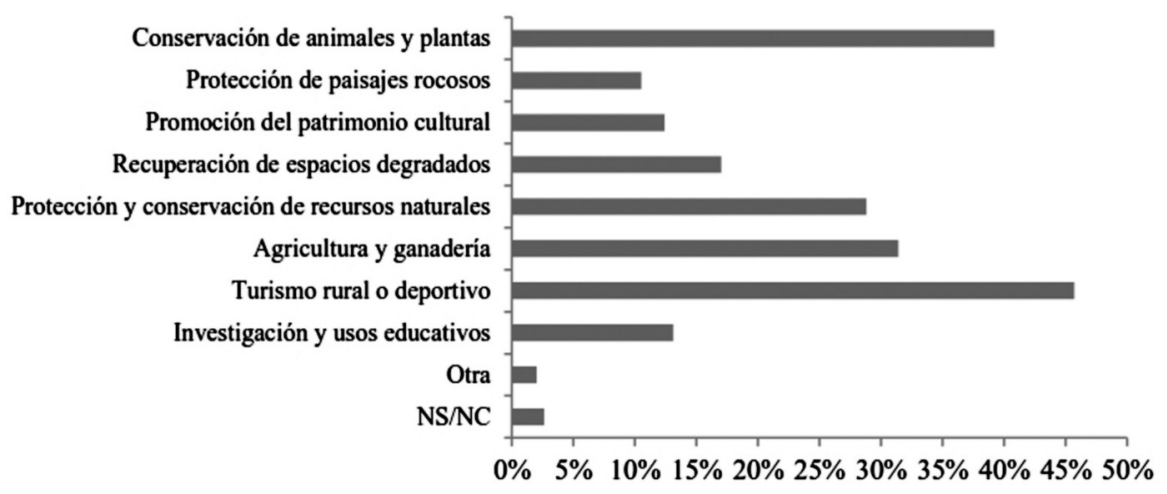

Estudios Geográficos, Vol. LXXVIII, 282, pp. 31-69, enero-junio 2017 ISSN: 0014-1496, eISSN: 1988-8546, doi: 10.3989/estgeogr.201702 


\section{Implicación poblacional}

Las preguntas incluidas en este bloque estaban orientadas hacia futuros procesos de gestión participativa en el marco de una futura declaración de ENP. Por ello la pregunta principal estuvo dirigida hacia conocer el modo en que el encuestado podría influir más efectivamente en la gestión del medio natural de su municipio (fig. 5). Entre las respuestas, un 26,8\% quisiera influir más activamente a través de consulta por voto para las propuestas y un mismo porcentaje apoyó la participación a través de asambleas abiertas. Destaca no obstante un muy relevante $24,9 \%$ que optó por la opción NSNC.

FIGURA 5

\section{VÍAS PARA LA PARTICIPACIÓN}

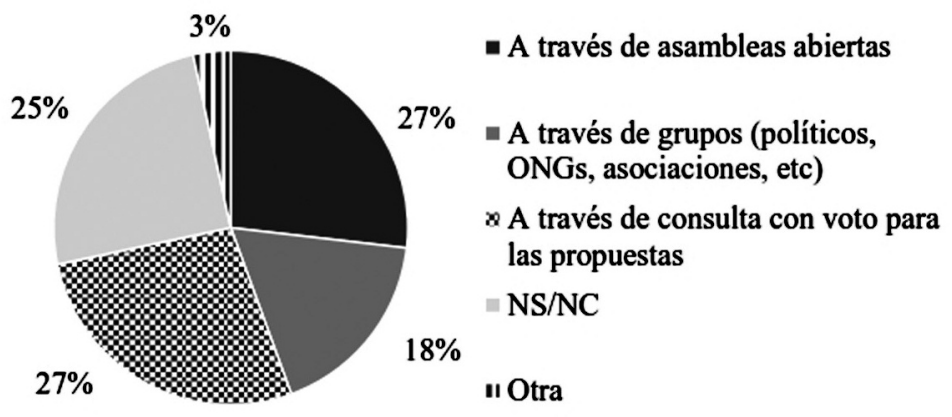

Al analizar la posible relación entre esta y otras variables sociodemográficas, resalta la vinculación positiva con el sector de actividad. El sector primario tuvo un apoyo mayoritario (33,3\% de los encuestados del sector) a las asambleas abiertas, mientras que en el sector industrial y de la construcción dominó la opción de representación a través de grupos (34,4\%). Finalmente, en el sector servicios la consulta a través de voto fue la preferida (37\%). A pesar de no haber sido identificadas otras relaciones, se detectó una mayor preferencia por la opción de la asamblea entre aquellos con menor nivel educativo, dominando la consulta por voto entre aquellos con un nivel educativo medio y la representación por grupos en un nivel alto de formación.

Entre las vías de acceso a la información proporcionada por el gobierno municipal, un 47,5\% indicó que le llegaba por el 'boca a boca', seguido por un $28,7 \%$ que obtenía la información a través del tablón del Ayuntamiento. Por otro lado, fue también evaluado el sentimiento de pertenencia a través de la 
disponibilidad a mudanza de residencia. Un $69 \%$ viviría en el mismo municipio y un $20,3 \%$ lo abandonaría tan solo a cambio de una mejora económica, indicando esto un fuerte sentimiento de arraigo.

Finalmente, la pertenencia a asociaciones mostró que un 53,3\% de los encuestados no estaba asociado a ningún colectivo y que dentro de los que sí lo hacían, dominaba la vinculación a asociaciones relacionadas con la actividad agraria.

\section{DISCUSIÓN DE LOS RESULTADOS Y PROPUESTA DE CONSERVACIÓN}

Tras 27 años de aplicación en Andalucía de la Ley-Inventario de Espacios Naturales Protegidos (1989-2016), el conocimiento en el medio rural de los ENPs es muy alto, de ahí que la ESA constituya una potente herramienta de trabajo para establecer un diagnóstico sobre el estado de opinión de las poblaciones y actores locales en relación con las políticas de declaración de ENPs y el posterior modelo de gestión participativa.

\section{La importancia de la NCS en el medio rural}

Entendiendo que la estabilidad de un espacio protegido depende en gran medida de la integración poblacional y la atención a sus problemas más inmediatos (Troitiño Vinuesa et al., 2005), los resultados de la ESA en la campiña alta del Subbético en el límite interprovincial de Sevilla-Cádiz, en un entorno con una consolidada presencia de ENPs, muestran que existe una asociación de ideas entre políticas de conservación y actividades económicas reguladas o adaptadas, o no, a las necesidades locales.

Ello tiene tanto más valor cuanto que la población local encuestada tiene como principal preocupación socio-económica «la escasez de empleo» (92\%) y «la dependencia al subsidio agrario» $(65,1 \%)$. O sea, se trata de una población que piensa en la bondad de las formas alternativas de desarrollo económico, sobre todo «turismo rural» $(44,1 \%)$ e «industria agroalimentaria» $(43,3 \%)$, ambas opciones derivadas de los recursos locales. En tal sentido son especialmente eficaces los ejemplos cercanos de desarrollo endógeno del Parque Natural de Grazalema y la aplicación de controles con marcas de calidad, todo ello adaptado a los nuevos requerimientos de la sociedad global y europea.

De manera complementaria, los resultados de la ESA muestran en general similitudes con otros sondeos de demandas sociales de la población rural en 
España que debemos resaltar (Fernández Muñoz, 2008; Rescia et al., 2008; Rico González y Gómez-Limón Rodríguez, 2012; Segrelles Serrano, 2013). Nos referimos a las preferencias que se detectan entre los encuestados, sea la que fuere la propuesta de planificación territorial, por: 1) el mantenimiento del medio rural como un territorio multifuncional (por ejemplo, cuando se apuesta por el mantenimiento de funciones de conservación de fauna y flora, $39,5 \%$; acciones agropecuarias, 31,6\%; o de protección y conservación de recursos naturales, $28,9 \%)$; y 2) por el uso de medidas proactivas en el territorio, tales como la reforestación o mejora de la gestión forestal (51\%), o la acción directa para el mantenimiento de las vías pecuarias $(49,8 \%)$, ambas propuestas entendidas como favorecedoras de un desarrollo territorial que mantiene la función social generadora de empleos locales.

$\mathrm{Si}$, como sabemos, la preocupación de la población por sus recursos locales y el interés por la calidad medioambiental de su entorno, constituyen dos claves de actualidad en la gestión de los territorios (Cruz Gallach, 2008), no menos relevantes son los problemas derivados de la conservación como resultado de la falta de comunicación entre las necesidades locales y las autoridades responsables (Bartos y Cihar, 2011).

En ambos casos resulta evidente el interés de focalizar la gestión del territorio en función de la solución de aquellos problemas. Como ya expusimos líneas atrás, la búsqueda de cauces para la resolución de los conflictos ha sido una de las estrategias del nuevo paradigma de la conservación (Reservas de Biosfera, Categoría V de UICN, propuestas de ecodesarrollo, integración de los paisajes antropizados y valores éticos) (Díaz del Olmo y Molina Vázquez, 1985; Goeghegan y Renard, 2002; Locke y Dearden, 2005; Troitiño Vinuesa et al., 2005; Zimmerer, 2006; Minteer y Miller, 2011; Sarkar y Montoya, 2011; Soulé, 2013), de ahí que los futuros ENPs deban reforzar los nuevos planteamientos auspiciados por la denominada NCS (Mace, 2014). Diversos ejemplos muestran cómo la creciente demanda social de valores de los ENPs actúan como dinamizadores territoriales, tal que los de la Comunidad de Cantabria (Delgado Viñas, 2012) o el humedal con gestión comunal agropecuaria del Parque Natural Agrario "Los Carrizales" en Elche (Segrelles Serrano, 2013).

El área de estudio, cuya población local ofrece un alto sentimiento de pertenencia y un conocimiento aceptable de su entorno natural, lógicamente más bajo en la franja más joven y más alto entre los propietarios de tierras, se convierte así pues en un excelente laboratorio geográfico donde promocionar un ENP renovado con planteamientos de uso y gestión concordantes con los principios de la NCS. 


\section{Parque Natural o Paisaje Protegido: una propuesta de integración y participación pública}

Interpretando los datos de la ESA, y teniendo presente las experiencias regionales, sub-regionales, nacionales e internacionales, la aplicación de una figura de conservación in situ en el área de estudio, debe pasar por un modelo integral de sostenibilidad social del tipo Categoría V de la UICN, en el que primen, junto a las directrices de conservación del patrimonio natural, las de fomento de las actividades socio-económicas existentes, específicamente las conducentes al mantenimiento de tres grandes líneas de actuación tendente a potenciar los bienes y servicios de sectores económicos de desarrollo endógeno: sistema agropecuario, las particulares del turismo rural y las de valorización del patrimonio cultural e histórico local.

En la legislación andaluza dicho modelo se concentra, con ciertas variaciones, en la figura de Parque Natural, la cual junto a la figura de Parque Nacional (Categoría II), son posiblemente las dos más extendidas y reconocidas socialmente como ENP. Se trata la de Parque Natural de una figura que fija su interés en la promoción de un desarrollo territorial sostenible en las áreas poco transformadas con núcleos de población insertos y valores ambientales de calidad (Ley 4/1989). Más de tres décadas desde las primeras declaraciones, la implantación del Parque Natural ha conllevado en el área de estudio (p.ej. Parques de Grazalema y Sierra de las Nieves) y fuera de él, algunos efectos indeseables de naturbanización en respuesta al aumento de usos residenciales en torno a espacios de calidad ambiental (Prados Velasco, 2012), al socaire del boom urbanístico de años anteriores (Delgado Viñas, 2008). Esta circunstancia no implica que la Categoría V haya perdido su interés como promotora de nuevas formas de desarrollo sostenible, ya que, junto a los efectos indeseables, existen numerosas muestras de dinamización territorial, algunas apoyadas con iniciativas de la UE (LEADER I y II), que se han concretado en nuevas alternativas económicas, como son las marcas de calidad de productos, servicios y denominaciones de origen asociadas a los territorios protegidos.

Sin embargo dos aspectos hacen disfuncional la aplicación de la figura de Parque Natural en este ámbito rural: 1) la demanda social de participación en la gestión, y 2) el fracaso de anteriores tentativas a nivel regional y sub-regional de aplicación de esta iniciativa.

La primera cuestión se desprende de los datos emanados de la ESA. Un 54\% de los encuestados se mostraron a favor de influir de forma activa, mediante sistema de votación o asambleas, en la gestión del territorio, porcentaje no muy alto pero en todo acorde con la tradición del asociacionismo local 
(41,7\% de los encuestados), fundamentalmente concentrado en organizaciones agrarias. Dado que la participación en los Parques Naturales se lleva a cabo a través de las Juntas Rectoras de sus Patronatos, donde los actores locales están representados por los ayuntamientos y ONGs, no parece que a través de ella se pueda alcanzar el modelo demandado de gestión participativa abierta y directa en la gestión del territorio. Como colofón, la desconfianza frente a este modelo de gestión medioambiental impulsado por la administración pública, se detectó ampliamente durante el proceso de encuestación.

En cuanto al segundo aspecto, la disfuncionalidad recae en la actitud del gobierno andaluz frente a la declaración de nuevos Parques Naturales, que en los últimos tres lustros parece haber abandonado la renovación del modelo de conservación y sostenibilidad de esta iniciativa, tras del impulso inicial del ecodesarrollo de los años ' 80 y ' 90 . Varios ejemplos de propuestas fallidas con similares valores ambientales en las proximidades del área de estudio podrían traerse a colación, pero baste recordar el rechazo de la propuesta sub-regional del Parque Natural del Genal en la Serranía de Ronda (Málaga), impulsado por diferentes colectivos sociales (Gómez Moreno, et al., 1998; Castillo Rodríguez, 2002) y tramitado con el apoyo de la Comisión de Medio Ambiente del Parlamento de Andalucía (El País, 9 de Junio de 2002), para comprender el enfoque reduccionista y poco renovador respecto de esta figura en las políticas medioambientales de los diferentes gobiernos de la Junta de Andalucía durante el siglo XXI.

Excluida la alternativa de Parque Natural, queda apelar a la figura de Paisaje Protegido, categoría homónima de la UICN, igualmente presente en la Ley andaluza 4/1989. Se aplica a lugares representativos para la conservación integral de paisajes terrestres (y marinos) (integración tradicional mediohombre, natural-cultural), con propuestas de mantenimiento de los asentamientos y procesos de usos tradicionales, turismo, investigación, seguimiento y educación ambiental. En España su aplicación tiene una larga tradición, ya que existe la figura desde el periodo pre-constitucional, habiéndose mantenido en las legislaciones autonómicas posteriores, e incluso reconocido en la firma del Convenio Europeo del Paisaje en 2000 (Mulero Mendigorri, 2013). Las dificultades de aplicación estriban en la diversidad de criterios utilizados para su puesta en valor, sobre todo en comparación con las demás figuras. La Comunidad de Canarias tiene una variedad de Paisajes Protegidos declarados por la calidad de sus valores naturales, estéticos y culturales (Mulero Mendigorri, 2013), y la de Asturias, por ejemplo, las mantiene para los paisajes de las cuencas mineras del Carbonífero favoreciendo la conservación de la geodiversidad local y la promoción turística de los georrecursos (Domínguez 
Cuesta y Jiménez Sánchez, 2009; Carcavilla et al., 2009). En la mayoría de ellos el instrumento de gestión es un Plan Especial que ejecuta la administración autonómica con sus peculiaridades específicas. En Andalucía la aplicación del Paisaje Protegido en la RENPA tiene un sentido finalista de corredor ecológico fluvial (Guadiamar y Rio Tinto), al objeto de desarrollar acciones encaminadas a la restauración ambiental. Existe pues una clara divergencia entre el objetivo de la figura y los de su aplicación. No obstante el interés de la categoría permanece, ya que su implantación podría configurar un modelo de gestión diferenciado de la figura de Parque Natural.

Una declaración de Paisaje Protegido del área de estudio como enclave geográfico natural de calidad que, además cuenta con la posibilidad de apoyar su declaración sobre la delimitación de la subcuenca fluvial del curso alto del río Guadaíra (tipo Categoría V de la UICN), permitiría adaptar un nuevo modelo de gestión participativa con instrumentos de acción directa en las tomas de decisiones. Tres grandes indicadores avalan esta propuesta territorial:

1) La existencia de paisajes relevantes con alto valor patrimonial, natural y cultural, susceptibles de alimentar nuevas propuestas para la Lista del Patrimonio Mundial de la UNESCO (Fernández Salinas y Silva Pérez, 2015), en un entorno geográfico con ciudades y asentamientos pequeños y medianos que potencian el marco agro-rural, como por ejemplo en el Parque Natural de la Sierra Norte de Sevilla y Reserva de la Biosfera de las Dehesas de Sierra Morena (Silva Pérez, 2014).

2) El mantenimiento de la multifuncionalidad económica con predominio de los usos rurales, el asociacionismo y las economías colaborativas consolidadas en la comarca, que permiten integrar la aplicación de las políticas de desarrollo rural de la PAC, así como la aplicación de la Ley de la Dehesa en Andalucía (2010) y la del Olivar (2011).

3) La implicación social de los municipios en la gestión participativa y proactiva del territorio, reforzando las funciones de ordenación de los recursos, la producción agrícola, de servicios y de conservación de la naturaleza, base del empleo estable o temporal en el medio rural.

\section{CONCLUSIONES}

En la actualidad las políticas de conservación de la naturaleza que tienen como finalidad la declaración de un ENP (conservación in situ), tienen como objetivo, junto a la conservación del patrimonio natural, la integración poblacional, el fomento de los valores y la sostenibilidad social. Son los nuevos 
planteamientos de la denominada NCS simplificados en la expresión nature and people que implica un renovado modelo de gestión participativa antes y después de una declaración de ENP.

Para poder llevar a cabo este modelo, la capacidad prospectiva de la ESA la convierte en una potente herramienta de trabajo al objeto de diagnosticar la opinión y actitud de las poblaciones y actores locales frente a la declaración y gestión posterior.

La aplicación a un enclave de la campiña alta del Subbético, en el límite de las provincias de Sevilla y Cádiz, en un entorno rural con abundante presencia cercana de ENPs y con una población dedicada prioritariamente al sector primario y de servicios, cuya principal preocupación socio-económica es la «escasez de empleo», pone de manifiesto el interés en las propuestas de gestión participativa. Así como, en la adecuación entre la figura de ENP y la regulación de actividades. Las preferencias locales se orientan hacia el mantenimiento de dos valores socio-económicos con gran reflejo en la calidad ambiental del paisaje: la multifuncionalidad rural y las medidas proactivas en el territorio local. Con estos resultados se propone la aplicación de la figura de Paisaje Protegido de la RENPA sin objetivo de restauración ambiental, sino como una propuesta tipo Categoría V de la UICN, que fomente el mantenimiento de las actividades socio-económicas que han determinados los paisajes rurales actuales, pero con una gestión participativa abierta a la población local, con base en la estructura municipal y diferenciada pues del modelo de Juntas Rectoras establecido para la figura de Parque Natural.

\section{AgradeCimientos}

Este trabajo, que ha sido financiado parcialmente por el Vicerrectorado de Relaciones Institucionales de la Universidad de Sevilla, es una contribución a los proyectos de conservación del patrimonio natural e histórico del Aula Miguel Cala Sánchez. Se agradece también el apoyo recibido por el Departamento de Geografía Física y Análisis Geográfico Regional de la Universidad de Sevilla en el trabajo de campo..

\section{BIBLIOGRAFÍA}

Abakerli, S. (2001): "A critique of development and conservation policies in environmentally sensitive regions in Brazil", Geoforum, 32, pp. 551-565.

Barber, C. V., Miller, K. y Boness, M. (eds.) (2004): Securing Protected Areas in the Face of Global Change: Issues and Strategies, Gland y Cambridge, IUCN, 234 pp. 
Bartos, L. y Cihar, M. (2011): "Socio Environmental Attitudes amongst the Inhabitants of Border Mountain Regions Close to the Former Iron Curtain: The Situation in the Czech Republic", Journal of Environmental Protection, 2, pp. 609-619.

Carcavilla, L., Durán, J. J., García-Cortés, A. y López Martínez, J. (2009): "Geological Heritage and Geoconservation in Spain: Past, Present and Future", Geoheritage, 1, pp. 75-91.

Castillo Rodríguez, J. A. (2002): El valle del Genal: paisajes, usos y formas de vida campesina, Málaga, Servicio de Publicaciones (CEDMA), Diputación Provincial de Málaga, 498 pp.

Colón, M. y Díaz del Olmo, F. (1989): Naturaleza en armonía. Espacios naturales en Andalucía, Sevilla, Ed. Alfar, 160 pp.

Cruz Gallach, H. (2008): "Conflictos territoriales y movilizaciones ciudadanas: algunas reflexiones sobre las formas de gobernanza territoriales actuales", Boletín de la Asociación de Geógrafos Españoles, 48, pp. 375-387.

Delgado Viñas, C. (2008): “Urbanización sin fronteras'. El acoso urbanístico a los espacios naturales protegidos", Boletín de la Asociación de Geógrafos Españoles, 47, pp. 271-310.

Delgado Viñas, C. (2012): "Protección y ordenación del medio natural en Cantabria. Normativa, instrumentos y conflictos territoriales", Boletín de la Asociación de Geógrafos Españoles, 60, pp. 115-140.

Díaz del Olmo, F. y Molina Vázquez, F. (1985): "Parques naturales andaluces: una estrategia de conservación y ecodesarrollo en regiones deprimidas", Revista de Estudios Andaluces, 4, pp. 147-156.

Domínguez Cuesta, M. J. y Jiménez Sánchez, M. (2009): "El paisaje protegido de las Cuencas Mineras (Asturias). Marco geomorfológico", Cuaternario y Geomorfología, 23/3-4, pp. 83-93.

Fernández Muñoz, S. (2008): "Participación pública, gobierno del territorio y paisaje en la Comunidad de Madrid", Boletín de la Asociación de Geógrafos Españoles, 46, pp. 97-119.

Fernández Salinas, V. y Silva Pérez, R. (2015): “Criterios para la identificación y selección de paisajes españoles susceptibles de ser incluidos en la Lista del Patrimonio Mundial de UNESCO", Boletín de la Asociación de Geógrafos Españoles, 68, pp. 253-278.

Folke, C., Holling, C. S. y Perrings, C. (1996): "Biological Diversity, Ecosystems, and the Human Scale", Ecological Applications, 6, pp. 1018-1024.

Furze, B., De Lacy, T. y Birckhead, J. (1996): Culture, Conservation and Biodiversity: The Social Dimension of Linking Local Level Development and Conservation Through Protected Areas, Chichester, John Wiley \& Sons, 269 pp.

Galvin, M. y Haller, T. (eds.) (2008): People, protected areas and global change. Participatory conservation in Latin America, Africa, Asia and Europe, Bern, Geographica Bernensia, 560 pp.

Geoghegan, T. y Renard, Y. (2002): "Beyond community involvement: lessons from the insular Caribbean", Parks, 12/2, pp. 16-27. 
Gómez-Baggethun, E. (2009): "Perspectivas del conocimiento ecológico local ante el proceso de globalización", Papeles de relaciones ecosociales y cambio global, 107, pp. 57-67.

Gómez Moreno, M. L. (1998): El Genal apresado. Agua y planificación: ¿desarrollo sostenible o crecimiento ilimitado?, Bilbao, Bakeaz, 279 pp.

Gómez Vargas, F. J., Boada Juncà, M. y Sánchez Mateo, S. (2008): “Análisis de los procesos de cambio global: el caso del Robledal de Ridaura (Parque Natural del Montseny. Barcelona)", Boletín de la Asociación de Geógrafos Españoles, 47, pp. 125-141.

Gray, M. (2013): Geodiversity: Valuing and Conserving Abiotic Nature, 2a ed., Chichester, Wiley-Blackwell, 508 pp.

Guevara, S. y Laborde, J. (2008): "The Landscape Approach: Designing New Reserves for Protection of Biological and Cultural Diversity in Latin America", Environmental Ethics, 30, pp. 251-262.

Gurrutxaga San Vicente, M. y Lozano Valencia, P. (2009): "La integración de la conectividad ecológica en los instrumentos de ordenación y planificación territorial: una revisión", Boletín de la Asociación de Geógrafos Españoles, 49, pp. 45-66.

Lausche, B. (2011): Guidelines for Protected Areas Legislation, Gland, IUCN, 370 pp.

Locke, H. y Dearden, P. (2005): "Rethinking protected area categories and the new paradigm", Environmental Conservation, 32/1, pp. 1-10.

Mace, G. M. (2014): “Whose conservation?”, Science, 345, pp. 1558-1560.

MAGRAMA (2015): Borrador del Informe 2014 sobre el estado del Patrimonio Natural y de la Biodiversidad en España, Madrid, Ministerio de Agricultura, Alimentación y Medio Ambiente.

Mallarach, J., Morrison, J., Kothari, A., Sarmiento, F., Atauri, J. A. y Wishitemi, B. (2008): "In defense of protected landscapes. A reply to some criticisms of category V protected areas and suggestions for improvement", en N. Dudley y S. Stolton (eds.), Defining Protected Areas: An International conference in Almeria, Spain, Gland, UICN, pp. 31-37.

Martín-López, B., García-Llorente, M., Palomo, I. y Montes, C. (2011): "The conservation against development paradigm in protected areas: Valuation of ecosystem services in the Doñana social-ecological system (southwestern Spain)", Ecological Economics, 70, pp. 1481-1491.

Martino, D. (2005): "Unleashing the wild: response to Locke and Dearden's 'rethinking protected area categories"', Environmental Conservation, 3, pp. 195-196.

Miller, T., Minteer, B. A. y Malan, L. C. (2011): "The new conservation debate: The view from practical ethics", Biological Conservation, 144, pp. 948-957.

Minteer, B. A. y Miller, T. R. (2011): "The New Conservation Debate: Ethical foundation, strategic trade-offs, and policy opportunities", Biological Conservation, 144, pp. 945-947.

Morillo, C. y Gómez-Campo, C. (2000): “Conservation in Spain, 1980-2000”, Biological Conservation, 95, pp. 165-174. 
Mulero Mendigorri, A. (2013): "Significado y tratamiento del paisaje en las políticas de protección de espacios naturales en España”, Boletín de la Asociación de Geógrafos Españoles, 62, pp. 129-145.

Prados Velasco, M. J. (2012): "Naturbanización y patrones urbanos en los Parques Nacionales de Andalucía”, Boletín de la Asociación de Geógrafos Españoles, 60, pp. 19-44.

Quiroga, D. (2009): "Crafting nature: the Galapagos and the making and unmaking of a natural laboratory", Journal of Political Ecology, 16, pp. 123-140.

Rescia A. J., Pons, A., Lomba, I., Esteban, C. y Dover, J. W. (2008): "Reformulating the social-ecological system in a cultural rural mountain landscape in the Picos de Europa región (northern Spain)", Landscape and Urban Planning, 88, pp. 23-33.

Rico González, M. y Gómez-Limón Rodríguez, J. A. (2012): "Preferencias y percepciones sociales sobre la multifuncionalidad del medio rural en Castilla y León", Boletín de la Asociación de Geógrafos Españoles, 60, pp. 399-424.

Sarkar, S. y Montoya, M. (2011): "Beyond parks and reserves: The ethics and politics of conservation with a case study from Perú", Biological Conservation, 144, pp. 979-988.

Segrelles Serrano, J. A. (2013): “Conservación ambiental y aprovechamiento agropecuario: el caso del Parque Natural Agrario 'Los Carrizales' (Elche, Alicante)", Boletín de la Asociación de Geógrafos Españoles, 62, pp. 295-316.

Silva Pérez, R. (2014): "Caracterización de paisajes en pequeñas y medianas ciudades. Propuesta metodológica aplicada a Constantina (Sierra Norte de Sevilla)", Boletín de la Asociación de Geógrafos Españoles, 64, pp. 297-319.

Sosa, B., Canton, V. y Achkar, M. (2014): "Los espacios de conservación en la gestión territorial: Análisis del Parque Nacional Esteros de Farrapos e Islas del Río Uruguay", Estudios Geográficos, 75/276, pp. 385-393.

Soulé, M. (2013): "The New Conservaton", Conservation Biology, 27/5, pp. 895-897.

Toledo, V. M. (2005): "Repensar la conservación: ¿Áreas naturales protegidas o estrategia bioregional?", Gaceta Ecológica, 77, pp. 67-83.

Troitiño Vinuesa, M. A., Marcos García-Blanco, F. J., García Hernández, M., Río Lafuente, M. I., Carpio Martín, J., Calle Vaquero, M. y Abad Aragón, L. D. (2005): "Los espacios protegidos en España: significación e incidencia socioterritorial", Boletín de la Asociación de Geógrafos Españoles, 39, pp. 227-265.

Wallance, S., Perkins, H. C. y Dixon, J. E. (2011): "What is social sustainability? A clarification of concepts", Geoforum, 42, pp. 342-348.

Zimmerer, K. S. (2006): "Geographical Perspectives on Globalization and Environmental Issues: The Inner-Connections of Conservation, Agriculture and Livelihoods", en K. S. Zimmerer (coord.), Globalization and New Geographies of Conservation, Chicago, The University of Chicago Press, pp. 1-43.

Fecha de recepción: 13 de mayo de 2015.

Fecha de aceptación: 4 de abril de 2016. 
ANEXo I - TABLAS DE ANÁLISIS DE FRECUENCIAS

1. ¿Ha oído alguna vez hablar de desarrollo sostenible?

\begin{tabular}{lrc}
\hline & $\mathrm{n}$ & $\%$ \\
\hline $\mathrm{Si}$ & 158 & 60,5 \\
\hline No & 97 & 37,2 \\
\hline NS/NC & 6 & 2,3 \\
\hline Total & 261 & 100 \\
\hline
\end{tabular}

2. De las siguientes frases, ¿cuál le parece que lo define mejor?

\begin{tabular}{lccc}
\hline & $\mathrm{n}$ & $\%$ & $\begin{array}{c}\% \\
\text { (sobre 158) }\end{array}$ \\
\hline 0 & 103 & 39,5 & \\
\hline $\begin{array}{l}\text { Un crecimiento económico rápido y constante } \\
\text { en el tiempo }\end{array}$ & 3 & 1,1 & 1,9 \\
\hline $\begin{array}{l}\text { Un desarrollo económico apoyado en el bienes- } \\
\text { tar social y la conservación de la naturaleza }\end{array}$ & 125 & 47,9 & 79,1 \\
\hline $\begin{array}{l}\text { Un tipo de desarrollo económico para los Espa- } \\
\text { cios Naturales Protegidos }\end{array}$ & 23 & 8,8 & 14,5 \\
\hline Otra & 1 & 0,4 & 0,6 \\
\hline NS/NC & 6 & 2,3 & 3,8 \\
\hline Total & 261 & 100 & 100 \\
\hline
\end{tabular}

3. ¿Qué valor tiene para usted el medio natural de su municipio?

\begin{tabular}{lcc}
\hline & $\mathrm{n}$ & $\%$ \\
\hline Alto & 117 & 44,8 \\
\hline Medio & 83 & 31,8 \\
\hline Bajo & 38 & 14,6 \\
\hline NS/NC & 23 & 8,8 \\
\hline Total & 261 & 100 \\
\hline
\end{tabular}


4. ¿Ha oído hablar de Espacios Naturales Protegidos?

\begin{tabular}{lrc}
\hline & $\mathrm{n}$ & $\%$ \\
\hline $\mathrm{Si}$ & 240 & 91,9 \\
\hline No & 19 & 7,3 \\
\hline NS/NC & 2 & 0,8 \\
\hline Total & 261 & 100 \\
\hline
\end{tabular}

5. ¿Conoce alguno de estos lugares?

\begin{tabular}{lccc}
\hline & $\mathrm{n}$ & $\%$ & $\%$ (sobre 19) \\
\hline Grazalema & 17 & 6,5 & 89,5 \\
\hline Doñana & 17 & 6,5 & 89,5 \\
\hline Fuente de Piedra & 10 & 3,8 & 52,6 \\
\hline Zaframagón & 9 & 3,4 & 47,4 \\
\hline El Chaparro de la Vega & 7 & 2,7 & 36,8 \\
\hline Ninguno & 3 & 1,1 & 15,8 \\
\hline NS/NC & 0 & 0 & 0 \\
\hline
\end{tabular}

6. ¿Con cuál de las siguientes frases está más de acuerdo? Los espacios protegidos:

\begin{tabular}{lccc}
\hline & $\mathrm{n}$ & $\%$ & $\begin{array}{c}\% \\
\text { (sobre 240) }\end{array}$ \\
\hline 0 & 21 & 8 & \\
\hline $\begin{array}{l}\text { Establecen prohibiciones y limitan el desarrollo } \\
\text { económico para conservar la naturaleza }\end{array}$ & 49 & 18,8 & 20,4 \\
\hline $\begin{array}{l}\text { Impulsan una economía de menor impacto am- } \\
\text { biental y ofrecen nuevas fuentes de empleo }\end{array}$ & 51 & 19,5 & 21,2 \\
\hline $\begin{array}{l}\text { Regulan las actividades económicas y fomentan } \\
\text { la conservación de la naturaleza }\end{array}$ & 84 & 32,2 & 35 \\
\hline Otro & 16 & 6,1 & 6,7 \\
\hline NS/NC & 40 & 15,3 & 16,7 \\
\hline Total & 261 & 100 & 100 \\
\hline
\end{tabular}


7. ¿Conoce alguno de los siguientes Espacios Naturales Protegidos? ¿Sabría decir su categoría de protección?

\begin{tabular}{lrrc}
\hline & $\mathrm{n}$ & $\%$ & $\begin{array}{c}\% \\
\text { (sobre 240) }\end{array}$ \\
\hline Parque Natural Grazalema & 115 & 44,1 & 47,9 \\
\hline Parque Nacional Doñana & 102 & 39,1 & 42,5 \\
\hline Reserva Natural Fuente de Piedra & 49 & 18,8 & 20,4 \\
\hline Reserva Natural Zaframagón & 57 & 21,8 & 23,7 \\
\hline Monumento Natural El Chaparro de la Vega & 33 & 12,6 & 13,7 \\
\hline NS/NC & 7 & 2,7 & 2,9 \\
\hline
\end{tabular}

8. ¿Cree que todo su municipio o alguna parte del mismo debería ser protegido?

\begin{tabular}{lrrc}
\hline & $\mathrm{n}$ & $\%$ & \% (sobre 240) \\
\hline 0 & 21 & 8 & \\
\hline $\mathrm{Si}$ & 126 & 48,3 & 52,5 \\
\hline $\mathrm{No}$ & 61 & 23,4 & 25,4 \\
\hline Si condicionado & 26 & 10 & 10,8 \\
\hline NS/NC & 27 & 10,3 & 11,2 \\
\hline Total & 261 & 100 & 100 \\
\hline
\end{tabular}

9. Si su municipio fuera incluido en un Parque Natural ¿qué actividades cree que deberían potenciarse más?

\begin{tabular}{lrrr}
\hline & $\mathrm{n}$ & $\%$ & $\begin{array}{c}\% \\
\text { (sobre 152) }\end{array}$ \\
\hline Investigación y usos educativos & 20 & 7,7 & 13,2 \\
\hline Turismo rural o deportivo & 70 & 26,8 & 46 \\
\hline Agricultura y ganadería & 48 & 18,4 & 31,6 \\
\hline Protección y conservación de recursos naturales & 44 & 16,9 & 28,9 \\
\hline Recuperación de espacios degradados & 26 & 10 & 17,1 \\
\hline Promoción del patrimonio cultural & 19 & 7,3 & 12,5 \\
\hline Protección de paisajes rocosos & 16 & 6,1 & 10,5 \\
\hline Conservación de animales y plantas & 60 & 23 & 39,5 \\
\hline Otra & 3 & 1,1 & 2 \\
\hline NS/NC & 4 & 1,5 & 2,6 \\
\hline
\end{tabular}


10. ¿En qué medida cree que los siguientes aspectos sociales y económicos dificultan el desarrollo de su municipio?

\begin{tabular}{lcc}
\hline & $\mathrm{n}$ & $\%$ \\
\hline Pérdida de población & 117 & 44,8 \\
\hline Escasez de empleo & 240 & 92 \\
\hline Estacionalidad del empleo & 151 & 57,9 \\
\hline Dependencia del subsidio agrario & 170 & 65,1 \\
\hline Bajo precio de los productos agrarios & 168 & 64,4 \\
\hline Emigración estacional & 115 & 44,1 \\
\hline Dependencia de pocos sectores económicos & 133 & 51 \\
\hline Otra & 4 & 1,5 \\
\hline NS/NC & 0 & 0 \\
\hline
\end{tabular}

11. ¿Qué medidas sociales o económicas considera que serían más necesarias?

\begin{tabular}{lcc}
\hline & $\mathrm{n}$ & $\%$ \\
\hline Mayor industria agroalimentaria & 113 & 43,3 \\
\hline Impulso turismo rural & 115 & 44,1 \\
\hline Apoyo a las producciones con sello de calidad & 30 & 11,5 \\
\hline Incremento número cooperativas & 75 & 28,7 \\
\hline Apoyo creación empresas & 138 & 52,9 \\
\hline Fomento comercio y hostelería & 44 & 16,9 \\
\hline Impulso industria energías renovables & 90 & 34,5 \\
\hline Otra & 7 & 2,7 \\
\hline NS/NC & 9 & 3,4 \\
\hline
\end{tabular}


12. ¿Cuáles de los siguientes aspectos cree que son un problema para el medioambiente de su municipio?

\begin{tabular}{lcc}
\hline & $\mathrm{n}$ & $\%$ \\
\hline Pérdida suelos & 93 & 35,6 \\
\hline Incendios & 45 & 17,2 \\
\hline Abandono o falta limpieza monte & 123 & 47,1 \\
\hline Suciedad ríos por vertidos & 140 & 53,6 \\
\hline Mal estado o invasión vías pecuarias & 130 & 49,8 \\
\hline Mal estado o invasión cauce ríos & 131 & 50,2 \\
\hline Bajada productividad campos & 89 & 34,1 \\
\hline Otra & 4 & 1,5 \\
\hline NS/NC & 13 & 5 \\
\hline
\end{tabular}

13. ¿Qué medidas cree que serían más necesarias para mejorar el medio ambiente de su municipio?

\begin{tabular}{lrc}
\hline & $\mathrm{n}$ & $\%$ \\
\hline Reforestación y mejora monte & 133 & 51 \\
\hline Recuperación dehesas & 47 & 18 \\
\hline Mayor reutilización residuos agrícolas & 88 & 33,7 \\
\hline Apoyo agricultura ecológica & 125 & 47,9 \\
\hline Recuperación razas ganaderas autóctonas & 30 & 11,5 \\
\hline Delimitación y restauración cauces y vías pecuarias & 100 & 38,3 \\
\hline Puesta en marcha planta depuradora & 64 & 24,5 \\
\hline Otra & 4 & 1,5 \\
\hline NS/NC & 13 & 5 \\
\hline
\end{tabular}

14. ¿Cuál es su opinión sobre las subvenciones a la agricultura? Indique con qué frase está más de acuerdo.

\begin{tabular}{lcc}
\hline & $\mathrm{n}$ & $\%$ \\
\hline $\begin{array}{l}\text { Las subvenciones a la agricultura son muy positivas } \\
\text { y deberían mantenerse }\end{array}$ & 69 & 26,4 \\
\hline $\begin{array}{l}\text { Es más importante que se valore el producto agrario } \\
\text { a tener subvenciones }\end{array}$ & 117 & 44,8 \\
\hline Otra & 22 & 8,4 \\
\hline NS/NC & 53 & 20,3 \\
\hline Total & 261 & 100 \\
\hline
\end{tabular}


15. ¿Conoce algún programa o ayuda en la zona que tenga financiación europea?

\begin{tabular}{lrc}
\hline & $\mathrm{n}$ & $\%$ \\
\hline $\mathrm{Si}$ & 79 & 30,3 \\
\hline $\mathrm{No}$ & 141 & 54 \\
\hline $\mathrm{NS} / \mathrm{NC}$ & 41 & 15,7 \\
\hline Total & 261 & 100 \\
\hline
\end{tabular}

17. Si pudiera influir más activamente en la gestión del medio natural de su municipio, ¿cómo cree que sería más efectiva su participación?

\begin{tabular}{lccc}
\hline & $\mathrm{n}$ & $\%$ \\
\hline A través de asambleas abiertas & 70 & 26,8 \\
\hline A través de grupos (políticos, ONGs, asociaciones, etc) & 46 & 17,6 \\
\hline A través de consulta con voto para las propuestas & 71 & 27,2 \\
\hline Otra & 9 & 3,4 \\
\hline NS/NC & 65 & 24,9 \\
\hline Total & 261 & 100 \\
\hline
\end{tabular}

18. Cuándo tiene que obtener información del Ayuntamiento, ¿cómo le llega?

\begin{tabular}{lcc}
\hline & $\mathrm{n}$ & $\%$ \\
\hline Prensa & 41 & 15,7 \\
\hline Tablón Ayuntamiento & 75 & 28,7 \\
\hline Página web Ayuntamiento & 15 & 5,7 \\
\hline Vecinos & 124 & 47,5 \\
\hline Internet & 26 & 10 \\
\hline TV local & 60 & 23 \\
\hline Otra & 29 & 11,1 \\
\hline NS/NC & 9 & 3,4 \\
\hline
\end{tabular}


19. Si tuviera libertad para elegir, ¿dónde viviría?

\begin{tabular}{lcc}
\hline & $\mathrm{n}$ & $\%$ \\
\hline Viviría en el mismo municipio & 180 & 69 \\
\hline $\begin{array}{l}\text { Viviría en otro lugar solo si eso le permitiera una } \\
\text { mejora económica }\end{array}$ & 53 & 20,3 \\
\hline Viviría en otro lugar & 24 & 9,2 \\
\hline NS/NC & 4 & 1,5 \\
\hline Total & 261 & 100 \\
\hline
\end{tabular}

20. Edad.

\begin{tabular}{lrc}
\hline & $\mathrm{n}$ & $\%$ \\
\hline $20-34$ & 69 & 26,4 \\
\hline $35-50$ & 126 & 48,3 \\
\hline $51-64$ & 51 & 19,5 \\
\hline$>0=65$ & 15 & 5,7 \\
\hline Total & 261 & 100 \\
\hline
\end{tabular}

22. Lugar de nacimiento.

\begin{tabular}{lrc}
\hline & $\mathrm{n}$ & $\%$ \\
\hline Otra comarca andaluza & 8 & 3,1 \\
\hline Otra CCAA. & 10 & 3,8 \\
\hline Extranjero & 5 & 1,9 \\
\hline Municipio comarca & 238 & 91,2 \\
\hline Total & 261 & 100 \\
\hline 23. Tiempo de residencia en el municipio. & $\mathrm{n}$ & \\
\hline & 8 & 3,1 \\
\hline$<2$ años & 13 & 5 \\
\hline 2-9 años & 38 & 14,6 \\
\hline = o >10 años & 202 & 17,4 \\
\hline Toda la vida & 261 & 100 \\
\hline Total &
\end{tabular}


24. ¿Está actualmente ocupado?

\begin{tabular}{lrc}
\hline & $\mathrm{n}$ & $\%$ \\
\hline $\mathrm{Si}$ & 169 & 64,8 \\
\hline No & 92 & 35,2 \\
\hline Total & 261 & 100 \\
\hline
\end{tabular}

25. Indique causa de la no ocupación:

\begin{tabular}{lrrc}
\hline & $\mathrm{n}$ & $\%$ & \% (sobre 92) \\
\hline 0 & 169 & 64,8 & \\
\hline Inactivo & 10 & 3,8 & 10,9 \\
\hline Estudiante & 4 & 1,5 & 4,3 \\
\hline Desempleado & 49 & 18,8 & 53,3 \\
\hline Jubilación & 22 & 8,4 & 23,9 \\
\hline Incapacitación & 6 & 2,3 & 6,5 \\
\hline Otra & 1 & 0,4 & 1 \\
\hline Total & 261 & 100 & 100 \\
\hline
\end{tabular}

26. ¿Podría indicar su ocupación principal y situación profesional?

\begin{tabular}{lcc}
\hline & $\mathrm{n}$ & $\%$ \\
\hline Primario & 122 & 46,7 \\
\hline Construcción & 21 & 8 \\
\hline Industria & 13 & 5 \\
\hline Comercio u hostelería & 45 & 17,2 \\
\hline Otros servicios & 51 & 19,5 \\
\hline Empresario, profesional o trabajador autónomo empleador & 37 & 14,2 \\
\hline Empresario, profesional o trabajador autónomo NO empleador & 24 & 9,2 \\
\hline Asalariado, trabajador por cuenta ajena con carácter fijo o indefinido & 89 & 34,1 \\
\hline Asalariado, trabajador por cuenta ajena con carácter eventual o & 91 & 34,9 \\
temporal & 9 & 3,4 \\
\hline Miembro de cooperativa & 2 & 0,8 \\
\hline Ayuda familiar & 9 & 3,4 \\
\hline NS/NC & 261 & 100 \\
\hline Total & 91 \\
\hline
\end{tabular}


27. ¿Es propietario de tierras? ¿Podría especificar el $n^{\circ}$ de hectáreas?

\begin{tabular}{lrr}
\hline & $\mathrm{n}$ & $\%$ \\
\hline$<5$ ha & 43 & 16,5 \\
\hline $5-20$ ha & 24 & 9,2 \\
\hline $20-49$ ha & 7 & 2,7 \\
\hline$>$ o = 50 ha & 10 & 3,8 \\
\hline No es propietario & 172 & 65,9 \\
\hline NS/NC & 5 & 1,9 \\
\hline Total & 261 & 100 \\
\hline
\end{tabular}

28. ¿Ha realizado estudios? ¿Podría indicar el máximo nivel completado?

\begin{tabular}{lcc}
\hline & $\mathrm{n}$ & $\%$ \\
\hline$<5$ años & 33 & 12,6 \\
\hline$>\mathrm{o}=5$ años & 30 & 11,5 \\
\hline Graduado escolar & 99 & 37,9 \\
\hline Educación secundaria & 30 & 11,5 \\
\hline FP & 29 & 11,1 \\
\hline Universitaria & 35 & 13,4 \\
\hline Otra & 1 & 0,4 \\
\hline NS/NC & 4 & 1,5 \\
\hline Total & 261 & 100 \\
\hline
\end{tabular}

30. ¿Pertenece o colabora con alguno de los siguientes grupos colectivos?

\begin{tabular}{lcc}
\hline & $\mathrm{n}$ & $\%$ \\
\hline Grupo político & 17 & 6,5 \\
\hline Asociación vecinal & 12 & 4,6 \\
\hline ONG & 14 & 5,4 \\
\hline Asociación religiosa & 24 & 9,2 \\
\hline Asociación agraria & 46 & 17,6 \\
\hline Asociación cultural & 26 & 10 \\
\hline Ninguna & 139 & 53,3 \\
\hline Otra & 13 & 5 \\
\hline NS/NC & 9 & 3,4 \\
\hline
\end{tabular}


31. Sexo.

\begin{tabular}{lcc}
\hline & $\mathrm{n}$ & $\%$ \\
\hline Mujer & 64 & 24,5 \\
\hline Hombre & 197 & 75,5 \\
\hline Total & 261 & 100 \\
\hline
\end{tabular}




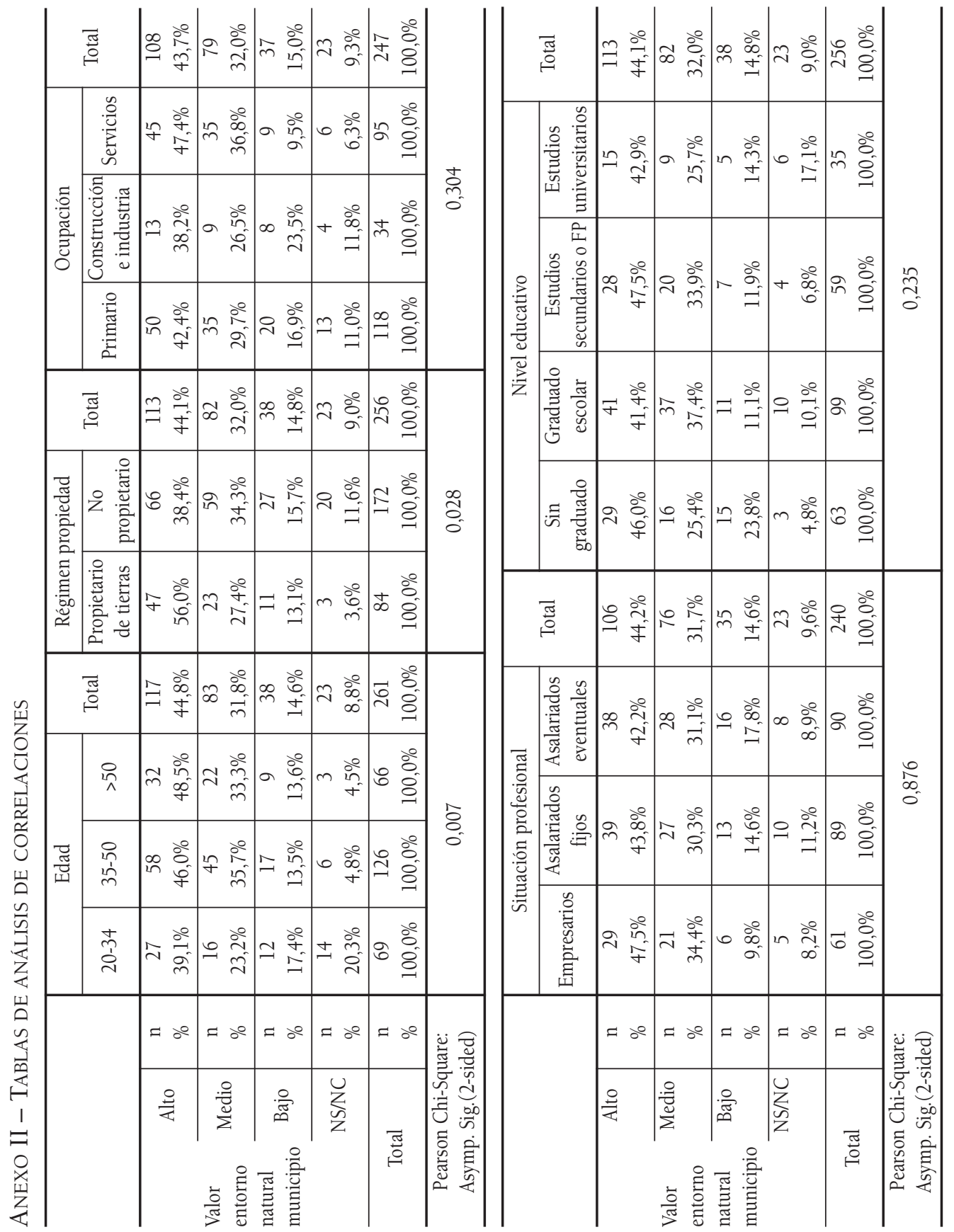

Estudios Geográficos, Vol. LXXVIII, 282, pp. 31-69, enero-junio 2017

ISSN: 0014-1496, eISSN: 1988-8546, doi: 10.3989/estgeogr.201702 


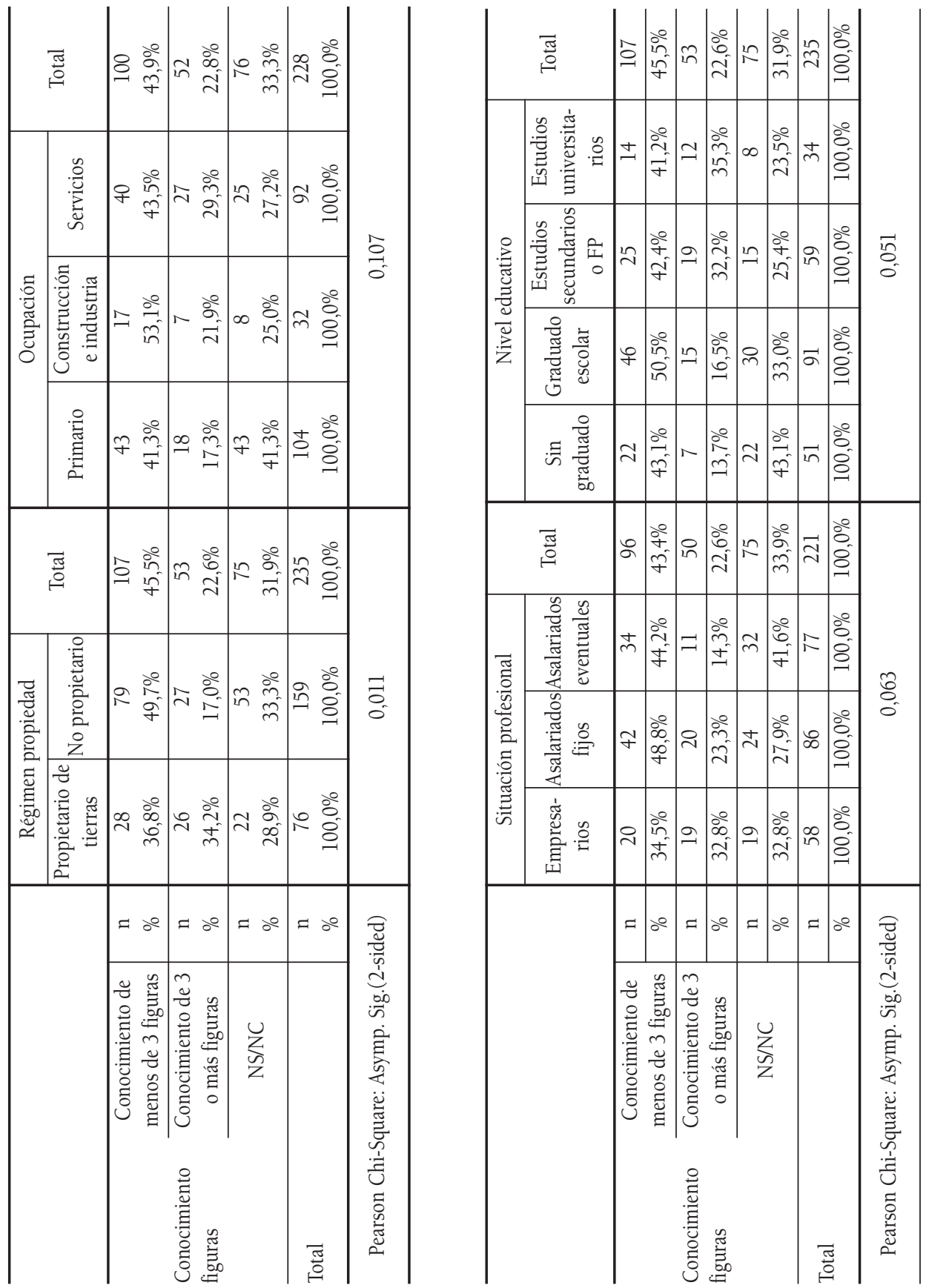

Estudios Geográficos, Vol. LXXVIII, 282, pp. 31-69, enero-junio 2017 ISSN: 0014-1496, eISSN: 1988-8546, doi: 10.3989/estgeogr.201702 


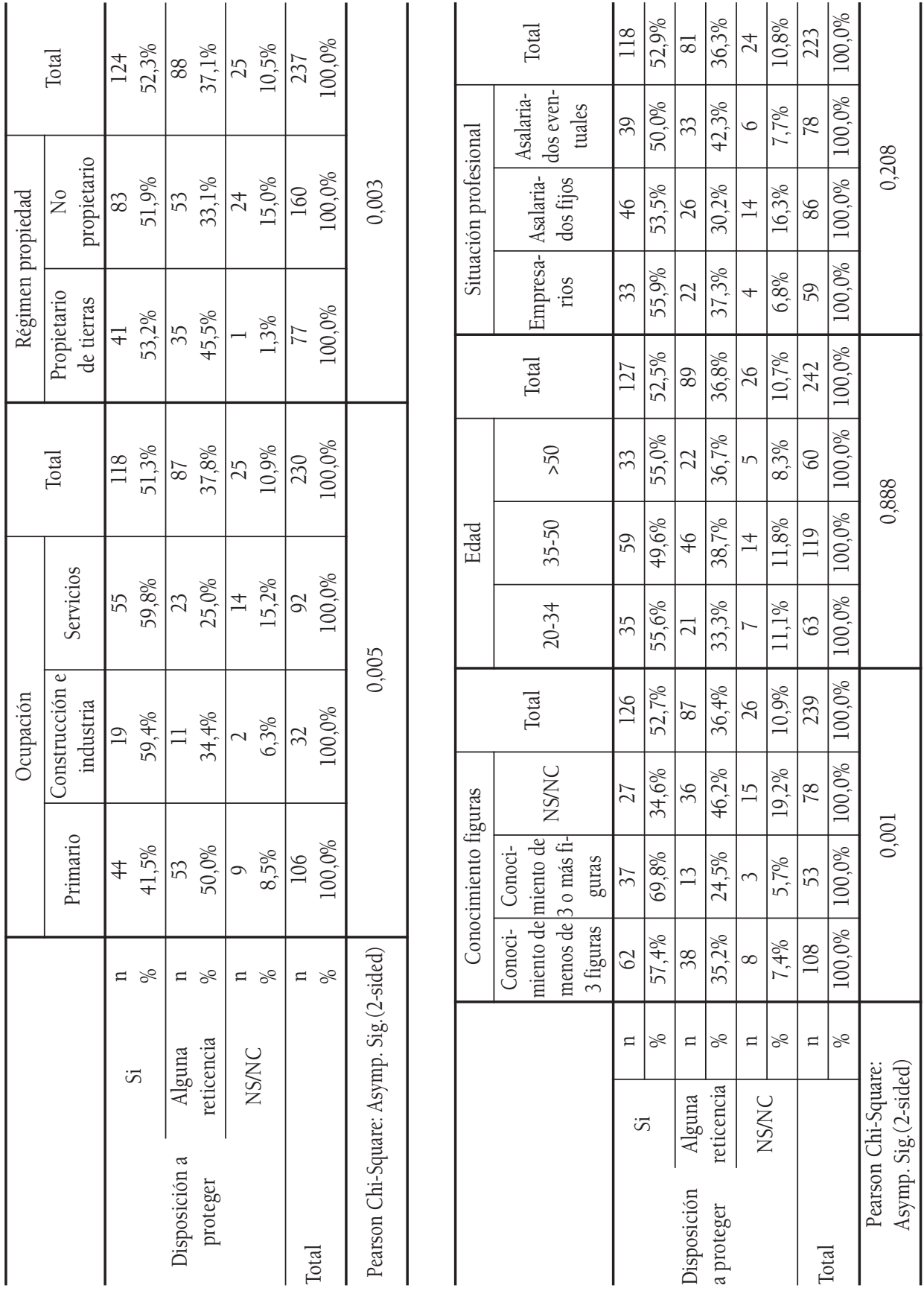

Estudios Geográficos, Vol. LXXVIII, 282, pp. 31-69, enero-junio 2017

ISSN: 0014-1496, eISSN: 1988-8546, doi: 10.3989/estgeogr.201702 


\begin{tabular}{|c|c|c|c|c|c|c|c|c|c|c|c|c|c|c|c|c|}
\hline & 氶 & 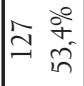 & $\infty \frac{8}{0}$ & 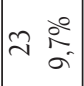 & $\mid \begin{array}{ll}\infty & 0 \\
\approx & 0 \\
0 & 8\end{array}$ & & & $\stackrel{\widetilde{\sigma}}{0}$ & & $\stackrel{\wp}{f}$ & 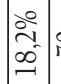 & $R \mid \begin{array}{l}\stackrel{0}{2} \\
\tilde{m}^{2} \\
\tilde{N}\end{array}$ & $\overleftarrow{b}$ & 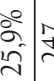 & $\begin{array}{l}+ \\
\dot{8} \\
\dot{8} \\
\dot{8}\end{array}$ & \\
\hline & 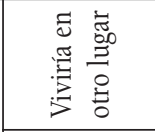 & $=\frac{8}{8}$ & $\infty \frac{\stackrel{\circ}{+}}{\mathrm{s}}$ & 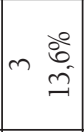 & 굥 & న & & 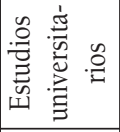 & $\ln \frac{\ddot{0}}{\tilde{I}}$ & $\approx$ & $\begin{array}{l}\stackrel{0}{0} \\
\tilde{m} \\
\tilde{m}\end{array}$ & $a \mid \begin{array}{l}0 \\
\frac{0}{2} \\
20\end{array}$ & $\sigma$ & 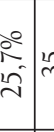 & $\hat{n} \begin{array}{l}0 \\
\dot{8} \\
\dot{8}\end{array}$ & \\
\hline 总 & 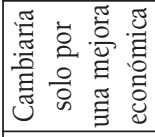 & $\bar{m} \underset{0}{0}$ & $\pm \underset{\substack{0 \\
0}}{0}$ & in $\begin{array}{l}0 \\
8 \\
0 \\
0\end{array}$ & in & 0 & 离 & 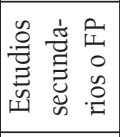 & $\stackrel{\infty}{\frac{8}{m}}$ & $\infty$ & 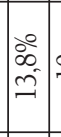 & 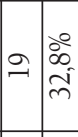 & $m$ & 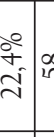 & 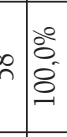 & $\begin{array}{l}\tilde{\sigma} \\
\tilde{\sigma} \\
0\end{array}$ \\
\hline & 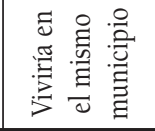 & $\infty \stackrel{\stackrel{\circ}{\check{n}}}{\stackrel{n}{n}}$ & ஃ & In & {$\left[\begin{array}{ll}0 & 8 \\
\hdashline & 8 \\
-1\end{array}\right.$} & & $\frac{\stackrel{\Xi}{\varpi}}{\stackrel{\Xi}{z}}$ & 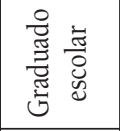 & 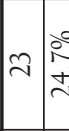 & $\stackrel{\infty}{-}$ & $\mid \begin{array}{c}0 \\
\stackrel{0}{+} \\
\stackrel{2}{2}\end{array}$ & 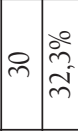 & $\approx$ & $\begin{array}{l}\stackrel{0}{2} \\
\vec{\lambda}\end{array}$ & $\hat{n}$ & \\
\hline & 氶 & 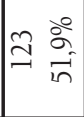 & $\infty \frac{\stackrel{\circ}{\stackrel{5}{m}}}{\infty}$ & $\stackrel{0}{\circ} \stackrel{0}{=}$ & \begin{tabular}{ll}
$\hat{\imath}$ & 8 \\
\hdashline & 8 \\
\end{tabular} & & & 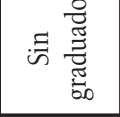 & ป & $r$ & $\stackrel{2}{\stackrel{2}{=}}$ & 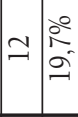 & 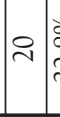 & 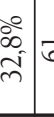 & $\begin{array}{l}-1 \\
0 \\
\dot{8} \\
\dot{8}\end{array}$ & \\
\hline & 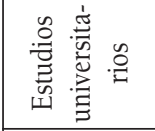 & 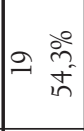 & $\exists \frac{\stackrel{\circ}{\vec{m}}}{m}$ & 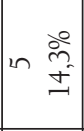 & 용 & & & 苂 & $8 \mid \begin{array}{l}\stackrel{0}{2} \\
1\end{array}$ & in & $\mid \begin{array}{l}\AA_{0}^{\circ} \\
\infty \\
0 \\
-1\end{array}$ & 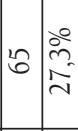 & $\widetilde{\sigma}$ & 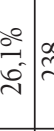 & 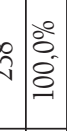 & \\
\hline 芯 & 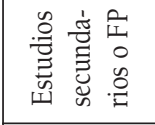 & م & $\approx \underset{m}{\stackrel{m}{m}}$ & $\sim \stackrel{\circ}{\stackrel{\Xi}{=}}$ & in $\begin{array}{l}0 \\
0 \\
8 \\
0\end{array}$ & 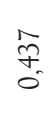 & & : & & $\stackrel{7}{ }$ & $\mid \begin{array}{l}0 \\
\stackrel{2}{2} \\
0\end{array}$ & 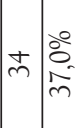 & 0 & 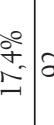 & V. & 6 \\
\hline$\frac{\overrightarrow{0}}{\vec{\Delta}}$ & 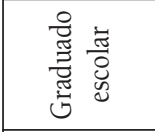 & a & ळi & 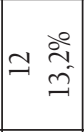 & न $\begin{array}{ll} & 8 \\
& 8 \\
8\end{array}$ & & 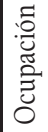 & 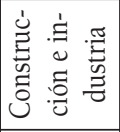 & in & $=$ & $\mid \begin{array}{l}\stackrel{0}{+} \\
\dot{+} \\
\dot{m} \\
\end{array}$ & $\infty \mid \begin{array}{l}80 \\
\stackrel{8}{2}\end{array}$ & $\infty$ & $\begin{array}{c}\overbrace{}^{\circ} \\
\overbrace{}^{-1}\end{array}$ & v & \\
\hline & $\approx \frac{\frac{0}{\tilde{T}}}{\tilde{E}}$ & 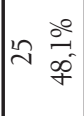 & $\cong \frac{\stackrel{\circ}{\curvearrowright}}{\frac{\infty}{+}}$ & $\sim \begin{array}{ll}\sim & \circ \\
\sim & \infty \\
& \infty\end{array}$ & in & & & 恚 & & 10 & $\begin{array}{l}\stackrel{0}{2} \\
\tilde{n}\end{array}$ & $\approx \mid \begin{array}{l}\stackrel{\partial}{\hat{\nu}} \\
\stackrel{\sim}{2}\end{array}$ & $\infty$ & $\begin{array}{l}\stackrel{0}{\circ} \\
\stackrel{m}{m}\end{array}$ & $=\mid \begin{array}{l}0 \\
8 \\
8 \\
8\end{array}$ & \\
\hline & & $=\circ^{\circ}$ & $\Rightarrow \circ$ & $=\circ^{\circ}$ & $\Rightarrow \circ$ & تֶ, & & & $\Rightarrow \circ$ & $\Rightarrow$ & $0^{\circ}$ & $\Rightarrow \circ$ & $\Rightarrow$ & $\therefore$ & $\Rightarrow \circ$ & 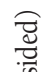 \\
\hline & & $\ddot{\sim}$ & 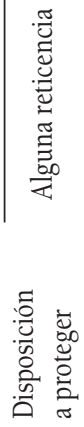 & $\sum_{\text {¿ }}^{u}$ & $\stackrel{\widetilde{3}}{0}$ & 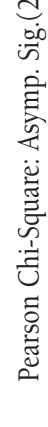 & & & 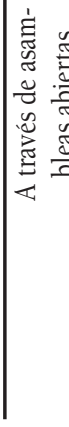 & & 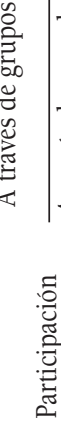 & 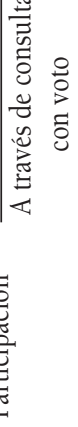 & $\sum_{z}^{z}$ & & 褐 & 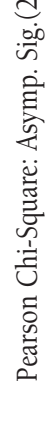 \\
\hline
\end{tabular}

Estudios Geográficos, Vol. LXXVIII, 282, pp. 31-69, enero-junio 2017 ISSN: 0014-1496, eISSN: 1988-8546, doi: 10.3989/estgeogr.201702 